\title{
Interval Observer versus Set-membership Approaches for Fault Detection in Uncertain Systems using Zonotopes
}

\author{
Masoud Pourasghar* | Vicenç Puig | Carlos Ocampo-Martinez
}

Automatic Control Department, Universitat Politècnica de Catalunya, Institut de Robòtica i Informàtica Industrial (CSIC-UPC), Supervision, Safety and Automatic Control Research Center (CS2AC), Barcelona, Spain

\section{Correspondence}

* Masoud Pourasghar, Automatic Control Department, Universitat Politècnica de Catalunya, Institut de Robòtica i Informàtica Industrial (CSIC-UPC), Supervision, Safety and Automatic Control Research Center (CS2AC).

Email: mpourasgharlaf@iri.upc.edu

\begin{abstract}
Summary
This paper presents both analysis and comparison of the interval-observer-based and set-membership approaches for the state estimation and fault detection (FD) in uncertain linear systems. The considered approaches assume that both state disturbance and measurement noise are modeled in a deterministic context following the unknown but bounded approach. The propagation of uncertainty in the state estimation is bounded through a zonotopic set representation. Both approaches have been mathematically related and compared when used for state estimation and FD. A case study based on a two-tanks system is employed for showing the relationship between both approaches while comparing their performance.
\end{abstract}

\section{KEYWORDS:}

uncertainty, interval observer, set membership, state estimation, fault detection, minimum detectable fault

\section{1 | INTRODUCTION}

Nowadays, engineering systems are becoming more and more complex and difficult to deal with. Faults affecting these systems may cause economic losses or even damage to human life, directly or indirectly. In order to keep safety in their operation and with the expected performance, several diagnosis and fault-tolerant control techniques have been proposed ${ }^{1}$. These techniques allow to know about the existence of a fault in the system and to activate mechanisms for compensating its effects as well as to cope with the effect of the disturbances and noises that are inevitable in practice.

Basically, fault diagnosis in dynamical systems is based on checking if the deviation of the system performance from its nominal behaviour can be due to the occurrence of a fault and it is not related to the disturbances (unknown inputs) and noises. Generally speaking, in a faulty dynamic system, first the the fault should be detected and isolated. Then, if needed, fault size can be estimated. Finally, the controller can be re-designed for adapting the closed-loop system to cope with the diagnosed fault. To this aim, fault-tolerant control (FTC) techniques have been developed for addressing the re-design of the controller for either decreasing or hiding the negative effects of faults on the system performance ${ }^{2}$.

On the other hand, the state estimation problem is an important topic in control theory since knowing the state of a system might be crucial for implementing many control and fault diagnosis methods. There are several approaches in the literature for estimating system states (and outputs). According to ${ }^{\sqrt[3]{3}}$, depending on the way uncertainties are modeled, state estimation approaches can be categorized into stochastic and deterministic approaches. In stochastic approaches, measurement noises and state disturbances are considered that can be modeled by some known statistical distribution (typically Gaussian). On the other hand, deterministic approaches assume noises and disturbances as unknown variables with known bounds. Among the family of deterministic approaches, interval observers and set-membership estimators have been introduced separately. The state estimation provided by both approaches is given in a form of a set of states at each time instant $\frac{4,5}{4}$. 
Interval observers have appeared during the last decade as an attractive approach for the state estimation of uncertain dynamical systems ${ }^{6178}$. In this approach, the state set can be estimated at time instant $k$ based on the state estimation set at time instant $k-1910$. Interval observers are also nowadays one of the ways for dealing with uncertainty in FD applications. A fault can be detected using an interval observer by generating an adaptive threshold for the residual signal that allow checking the consistency of the measurements with the estimated output. In other words, the existence of the fault can be detected by comparing the residual with a threshold value. In the case that the residual is larger than the threshold, the fault is detected. Otherwise, the effect of the fault cannot be detected since the fault effect is lower than the effect of the uncertainties. Likewise, the setmembership state estimation is an alternative approach that allows to estimate the system state including the measured output and bounded noises $\frac{1112}{12}$ by means of the intersection between the prediction state set and the set of states consistent with the output measurements (as it will be further explained in the following part of the paper) ${ }^{10}$. The set enclosing the state estimation considering uncertainty can be approximated by means of several geometrical shapes, e.g., polytopes ${ }^{13 / 14}$, ellipsoids ${ }^{15|16| 17 \mid 18}$ and zonotopes $19|20| 1121$.

A considerable amount of literature has also been published on a variety of approaches to increase the robustness of the FD methods such that the residual is obtained to be insensitive to uncertainty, while at the same time sensitive to fault ${ }^{[22[23] 24}$. This is the case of FD filters that are designed by considering the robustness against disturbances, noise or any uncertainties using, e.g., $H_{\infty}$ optimization, Linear Matrix Inequality (LMI) techniques ${ }^{2425}$. But, recent results show that the filter design technique considering only the rejection of the effect of uncertainties has a major drawback since the sensitivity to the fault needs to be considered in FD filter design ${ }^{26}$. This is the case of ${ }^{27}$ where the multi-objective FD design based on $H_{-} / H_{\infty}$ techniques is considered. Indeed, the worst-case of the fault sensitivity is taken into account by the smallest not a null singular value of the transfer function matrix from fault to residual at either $\omega=0$ or in given frequency range ${ }^{28 / 2930}$. Likewise, a significant amount of literature has been published discussing this multi-objective design optimization problem, e.g., $H_{\infty} / H_{\infty}, H_{2} / H_{\infty}$ or $H_{2} / H_{2}$ problems 2613132133 .

Furthermore, several model-based FD methods have been developed for computing the minimum magnitude of the fault that can be detected through the use of interval-observer-based and set-membership approaches. The difference between the modelbased approaches regarding the minimum detectable fault is related to the different ways of modeling the fault. A fault in a state estimation scheme can be modeled as a state or as an unknown input ${ }^{34}$. Additionally, the effect of observer gain and its influence on the computation of the minimum detectable fault has already been studied in 35 .

However, to the best of the authors knowledge, both interval-observer-based and set-membership approaches are still considered as two different approaches. Therefore, the main contribution of this paper is to analyze and compare both approaches in order to find out their relationship in mathematical terms when applied to state estimation and FD, and to establish the advantages and disadvantages of each approach. to find out their relationship in a formal mathematical framework. Moreover, the comparison is also performed in the context of FD application by means of proposing a novel FD test to connect the approaches for the characterization of the minimum detectable fault. Finally, the relationship between both approaches when applied to both the state estimation and FD is illustrated by using a two-tanks case study.

The remainder of this paper is organized as follows: first, the problem formulation regarding state estimation for each approach and the way to relate them is discussed in Section 2 Then, the approaches are compared into the FD application context in Section 3 . In Section 4 , a case study based on a two-tanks system is proposed in order to show the aforementioned relationships and present the comparison results. Finally, in Section 5 the main conclusions of the paper are drawn. For completeness, relevant definitions and properties of zonotopes are recalled in the Appendix.

\section{Notation}

Throughout this paper, $\mathbb{R}$ denotes the set of real numbers, $\mathbb{R}^{n}$ denotes the set of $n$-dimensional real numbers, $\mathbb{Z}_{+}$indicates the set of non-negative integer numbers, $\oplus$ denotes the Minkowski sum, the matrices are written using capital letter e.g., $A$, the calligraphic notation is used for presenting the sets, e.g., $\mathcal{X}, \mathbf{B}^{r}$ is a $r$-dimensional unitary box and the inequalities are interpreted element-wise, $\|\cdot\|_{s}$ denotes the $s$-norm, $\min$ and $\max$ are the operators that allow to obtain the minimum and the maximum value of a given interval, respectively, and $\square \mathcal{X}$ shows the interval hull of a set $\mathcal{X}$. 


\section{2 | STATE ESTIMATION}

\section{1 | System set-up}

This paper considers a linear uncertain discrete-time invariant system modeled as

$$
\begin{aligned}
x_{k+1} & =A x_{k}+B u_{k}+E_{\omega} \omega_{k}, \\
y_{k} & =C x_{k}+E_{\eta} \eta_{k},
\end{aligned}
$$

where $x_{k} \in \mathbb{R}^{n_{x}}$ is the state vector, $u_{k} \in \mathbb{R}^{n_{u}}$ and $y_{k} \in \mathbb{R}^{n_{y}}$ denote the input and the output vectors, respectively. The system matrices are $A \in \mathbb{R}^{n_{x} \times n_{x}}, B \in \mathbb{R}^{n_{x} \times n_{u}}$ and $C \in \mathbb{R}^{n_{y} \times n_{x}}$. The disturbance/noise distribution diagonal matrices are denoted by $E_{\omega}$ and $E_{\eta}$ with appropriate dimensions while $k \in \mathbb{N}$ indicates the discrete time. Moreover, $\omega_{k} \in \mathbb{R}^{n_{\omega}}$ denotes the state disturbance and $\eta_{k} \in \mathbb{R}^{n_{\eta}}$ represents the sensor noise, both assumed unknown but bounded, i.e.,

$$
\begin{aligned}
\mathcal{W} & =\left\{\omega_{k} \in \mathbb{R}^{n_{\omega}}:\left|\omega_{k}-\omega^{c}\right| \leq \bar{\omega}, \omega^{c} \in \mathbb{R}^{n_{\omega}}, \bar{\omega} \in \mathbb{R}^{n_{\omega}}\right\}, \\
\mathcal{V} & =\left\{\eta_{k} \in \mathbb{R}^{n_{\eta}}:\left|\eta_{k}-\eta^{c}\right| \leq \bar{\eta}, \eta^{c} \in \mathbb{R}^{n_{\eta}}, \bar{\eta} \in \mathbb{R}^{n_{\eta}}\right\},
\end{aligned}
$$

where $\omega^{c}, \bar{\omega}, \eta^{c}$ and $\bar{\eta}$ are constant vectors. Disturbance and noise bounds in (2) can be represented using a zonotopic representation of the $\operatorname{sen}^{1}$ as

$$
\begin{aligned}
\mathcal{W} & =\omega^{c} \oplus H_{\bar{\omega}} \mathbf{B}^{n_{\omega}}, \\
\mathcal{V} & =\eta^{c} \oplus H_{\bar{\eta}} \mathbf{B}^{n_{\eta}},
\end{aligned}
$$

where $H_{\bar{\omega}} \in \mathbb{R}^{n_{\omega} \times n_{\omega}}$ and $H_{\bar{\eta}} \in \mathbb{R}^{n_{\eta} \times n_{\eta}}$ are two non-zero diagonal matrices composed of $\bar{\omega}$ and $\bar{\eta}$ in their diagonal entries. Moreover, $\mathbf{B}^{n_{\omega}}$ and $\mathbf{B}^{n_{n}}$ are unitary boxes.

Remark 1. Noticed that the inequalities associated to $\left|\omega_{k}-\omega^{c}\right|$ and $\left|\eta_{k}-\eta^{c}\right|$ in (2) are considered component-wise.

Assumption 1. The pair $\{A, C\}$ is detectable.

Assumption 2. Additive uncertainties represented in (3) are assumed to be bounded by unitary hypercube zonotope centered at the origin. It means, $\forall k \geq 0, \omega=[-1,1]$, i.e., $\omega^{c}=\emptyset$ and $H_{\bar{\omega}}=I_{n_{\omega}}$. Moreover, $v=[-1,1]$, i.e., $\eta^{c}=\emptyset$ and $H_{\bar{\eta}}=I_{n_{\eta}}$, where $I_{n_{\omega}} \in \mathbb{R}^{n_{\omega} \times n_{\omega}}$ and $I_{n_{\eta}} \in \mathbb{R}^{n_{\eta} \times n_{\eta}}$ denote identity matrices.

For the sake of simplification, the acronyms IOA and SMA will be used to denote the interval-observer-based and set-membership approaches, respectively.

\section{2 | State estimation using SMA}

The state estimation in the case of SMA is carried out based on ${ }^{[10}$ and Proposition 1

Proposition 1. Considering the dynamical model (1), the center $\hat{x}^{\mathrm{c}_{\mathrm{sm}}}$ and the segment (shape) matrix $\hat{H}^{\mathrm{x}_{\mathrm{sm}}}$ of the state-bounding zonotope $\hat{\mathcal{X}}^{\mathrm{sm}}$ corrected by the $i^{\text {th }}$ output, i.e.,

$$
\hat{\mathcal{X}}_{k, i}^{\mathrm{sm}}=\hat{x}_{k}^{\mathrm{c}_{\mathrm{sm}}} \oplus \hat{H}_{k}^{\mathrm{x}_{\mathrm{sm}}} \mathbf{B}^{\mathrm{n}_{\mathrm{x}}+\mathrm{n}_{\omega}+1},
$$

can be obtained by intersecting the prediction state set $\mathcal{X}_{k}^{e}$ and the set of states consistent with each output strip $\mathcal{X}_{k, i}^{y k}$ as

$$
\begin{aligned}
\hat{x}_{k}^{\mathrm{c}_{\mathrm{sm}}} & =\hat{x}_{k}^{\mathrm{c}_{\mathrm{e}}}+\lambda_{k, i}\left(y_{k, i}-C_{i} \hat{x}_{k}^{\mathrm{c}_{\mathrm{e}}}\right), \\
\hat{H}_{k}^{\mathrm{x}_{\mathrm{sm}}} & =\left[\left(I-\lambda_{k, i} C_{i}\right) H_{k}^{\mathrm{x}_{\mathrm{e}}}-\lambda_{k, i} E_{\eta, i}\right],
\end{aligned}
$$

with

$$
\begin{aligned}
\hat{x}_{k}^{\mathrm{c}_{\mathrm{e}}} & =A \hat{x}_{k-1}^{\mathrm{c}_{\mathrm{sm}}}+B u_{k-1}, \\
H_{k}^{\mathrm{x}_{\mathrm{e}}} & =\left[\begin{array}{ll}
A \hat{H}_{k-1}^{\mathrm{x}_{\mathrm{sm}}} & E_{\omega}
\end{array}\right],
\end{aligned}
$$

where $\lambda_{i}$ is a vector that provides degrees of freedom to tune the system monitoring, e.g., optimizing the state-bounding zonotope to be as robust as possible with respect to effect of uncertainties. 


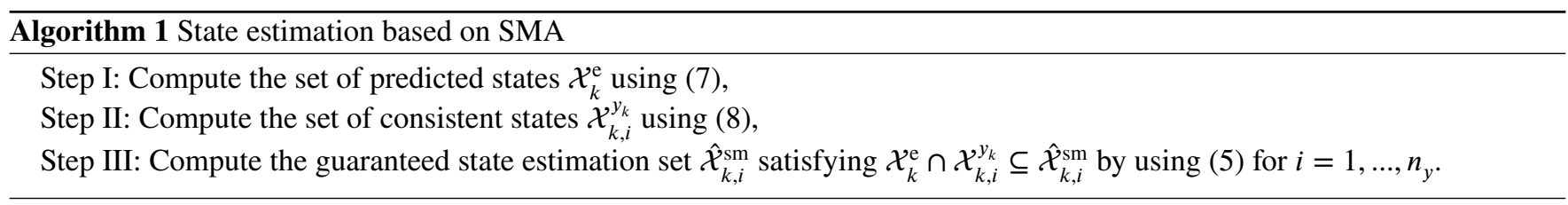

Proof. Considering the dynamical model (1), the prediction state set can be computed as a zonotope, i.e.,

$$
\mathcal{X}_{k}^{\mathrm{e}}=\hat{x}_{k}^{\mathrm{c}_{\mathrm{e}}} \oplus H_{k}^{\mathrm{x}_{\mathrm{e}}} \mathbf{B}^{\mathrm{n}_{\mathrm{x}}+\mathrm{n}_{\omega}},
$$

where $\hat{x}^{\mathrm{c}_{\mathrm{e}}}$ and $H^{\mathrm{x}_{\mathrm{e}}}$ denote the center and segments of the zonotope $\mathcal{X}^{\mathrm{e}}$, respectively. Furthermore, $\hat{x}^{\mathrm{c}_{\mathrm{e}}}$ and $H^{\mathrm{x}_{\mathrm{e}}}$ can be calculated using (6) at time instant $k$. Additionally, a strip $\mathcal{X}_{k}^{y_{k}}$ is computed by considering each measurement component $y_{k}$ as

$$
\mathcal{X}_{k, i}^{y_{k}}=\left\{x_{k} \in \mathbb{R}^{n_{x}}:\left|C_{i} x_{k}-y_{k, i}\right| \leq E_{\eta, i}\right\} .
$$

According to $\frac{10}{10}$, intersection between the zonotope in (7) and the obtained strip in (8) provides the state estimation using SMA corrected by each measurement. In order to compute the intersection between a zonotope and a strip, the Property A2 in Appendix can be used. Therefore, using Property A2 to compute the intersection of (7) and (8), the time evolution of the center and the segments of state-bounding zonotope (4) are given as it is derived in (5).

Remark 2. According to ${ }^{36}$, in order to apply Algorithm 1 to multi-output cases, i.e., $n_{y}>1$, the system can be considered as several single-output systems based on the dimension of the measurement $n_{y}$. Then, Algorithm 1 can be applied at each step to compute the intersection between the predicted state set and the obtained strip for each component of the measured output using Property A2. Considering the system as several single-output systems leads to use Property A2 towards computing the optimal vectors $\lambda_{1}, \lambda_{2}, \ldots, \lambda_{n_{y}}$, independently ${ }^{2}$

Based on (5), the obtained state-bounding zonotope using SMA is parametrized by means of a $\lambda$. Computing the parameter $\lambda$ can be done using several methods, e.g., singular value decomposition, segments minimization, volume minimization and the P-radius minimization $10 \mid 11$. In this paper, the optimal value of $\lambda$ is explicitly computed by using Theorem 1 .

Theorem 1. Considering the dynamical model (1) and the state-bounding zonotope (5) at time instant $k$, the optimal value of $\lambda$ that minimizes the F-radius of the zonotope (5) to be robust with respect to effect of uncertainties at time instant $k+1$ is computed as

$$
\lambda_{k, i}^{*}=\left(\hat{H}_{k}^{\mathrm{x}_{\mathrm{sm}}} \hat{H}_{k}^{\mathrm{x}_{\mathrm{sm}}{ }^{\top}} C_{i}^{\top}\right)\left(C_{i} \hat{H}_{k}^{\mathrm{x}_{\mathrm{sm}}} \hat{H}_{k}^{\mathrm{x}_{\mathrm{sm}}{ }^{\top}} C_{i}^{\top}+E_{\eta, i} E_{\eta, i}^{\top}\right)^{-1},
$$

where the superscript $*$ denotes the optimal value.

Proof. The proof follows from the results presented in 10 .

Finally, Algorithm 1 summarizes the state estimation procedure using SMA following the results presented in this section.

\section{3 | State estimation using IOA}

Monitoring the dynamical model (1) can be done by designing a Luenberger observer of the form

$$
\hat{x}_{k}^{\text {io }}=A \hat{x}_{k-1}^{\text {io }}+B u_{k-1}+L_{k}^{\text {io }}\left(y_{k-1}-C \hat{x}_{k-1}^{\text {io }}\right),
$$

where $\hat{x}^{\text {io }}$ is the state estimation using IOA. Moreover, the observer gain $L^{\text {io }}$ should be computed such that $\left(A-L^{\text {io }} C\right)$ is a Schur matrix.

Assumption 3. The initial state $x_{0}$ belongs to the zonotopic set $\hat{\mathcal{X}}_{0}^{\mathrm{io}}=\hat{x}_{0}^{\mathrm{c}_{\mathrm{io}}} \oplus \hat{H}_{0}^{\mathrm{x}_{\mathrm{io}}} \mathbf{B}^{\mathrm{n}_{\mathrm{x}_{\mathrm{io}}}}$, where $\hat{x}_{0}^{\mathrm{c}_{\mathrm{io}}} \in \mathbb{R}^{n_{x}}$ denotes the center and $\hat{H}_{0}^{\mathrm{x}_{\mathrm{io}}} \in \mathbb{R}^{n_{x} \times n_{R_{0}}}$ is a non-empty matrix containing the generators matrix of the initial zonotope $\hat{\mathcal{X}}_{0}^{\text {io }}$.

According to 19 , the resulting interval observer of the dynamical model (1) can be defined by considering the observer (10) and Assumptions 1 and 2 both $\hat{x}_{k}^{\mathrm{c}_{\mathrm{io}}}$ and $\hat{H}_{k}^{\mathrm{x}_{\mathrm{io}}}$ from the state-bounding zonotope $\hat{\mathcal{X}}_{k}^{\mathrm{x}_{\mathrm{io}}}$, i.e.,

$$
\hat{\mathcal{X}}_{k}^{\mathrm{io}}=\hat{x}_{k}^{\mathrm{c}_{\mathrm{io}}} \oplus \hat{H}_{k}^{\mathrm{x}_{\mathrm{io}}} \mathbf{B}^{\mathrm{n}_{\mathrm{x}}+\mathrm{n}_{\mathrm{\omega}}+1},
$$

\footnotetext{
${ }^{2}$ This approach called equivalent single-measurement approach in ${ }^{36}$
} 
are obtained as

$$
\begin{aligned}
\hat{x}_{k}^{\mathrm{c}_{\mathrm{io}}} & =\hat{p}_{k}^{\mathrm{c}_{\mathrm{io}}}+L_{k}^{\mathrm{io}}\left(y_{k-1}-C \hat{p}_{k}^{\mathrm{c}_{\mathrm{io}}}\right), \\
\hat{H}_{k}^{\mathrm{x}_{\mathrm{io}}} & =\left[\left(I-L_{k}^{\mathrm{io}} C\right) \hat{H}_{k}^{\hat{\mathrm{p}}^{\mathrm{io}}}-L_{k}^{\mathrm{io}} E_{\eta}\right],
\end{aligned}
$$

with

$$
\begin{aligned}
\hat{p}_{k}^{\mathrm{c}_{\mathrm{io}}} & =A \hat{x}_{k-1}^{\mathrm{c}_{\mathrm{io}}}+B u_{k-1}, \\
\hat{H}_{k}^{\hat{\mathrm{p}}^{\mathrm{io}}} & =\left[\begin{array}{ll}
A \bar{H}_{k-1}^{\mathrm{x}_{\mathrm{io}}} & E_{\omega}
\end{array}\right],
\end{aligned}
$$

where $\bar{H}^{\mathrm{x}_{\mathrm{io}}}=\downarrow_{q}\left\{\hat{H}^{\mathrm{x}_{\mathrm{io}}}\right\}$ (see Property A3 in Appendix). Furthermore, the state inclusion property holds by using the reduction operator for all $k \geq 0$.

$\mathrm{In}$ [37, an special type of IOA named Zonotopic Filter (ZF) is proposed. This approach can be viewed as the deterministic counterpart of the stochastic Kalman filter when uncertainties (noise and disturbance) are assumed to be unknown but bounded. The ZF yields the same zonotopic representations for the set in (12) for the state estimation of (1) but using the optimal observer gain given by Theorem 2

Theorem 2. Considering $x \in \hat{\mathcal{X}}^{\mathrm{io}}$ at time $k$, the optimal observer gain $L_{k}^{\mathrm{io}, *}$ that minimizes the F-radius of the state-bounding zonotope (11) at time instant $k+1$ is computed as

$$
L_{k}^{\mathrm{io}, *}=\left(A \hat{H}_{k}^{\mathrm{x}_{\mathrm{io}}} \hat{H}_{k}^{\mathrm{x}_{\mathrm{io}}{ }^{\top}} C^{\top}\right)\left(C \hat{H}_{k}^{\mathrm{x}_{\mathrm{io}}} \hat{H}_{k}^{\mathrm{x}_{\mathrm{io}}{ }^{\top}} C^{\top}+E_{\eta} E_{\eta}^{\top}\right)^{-1} .
$$

Proof. According to ${ }^{37}$, minimizing the $F$-radius and $F_{W}$-radius of a zonotope is equivalent to minimize the trace of its covariance. Therefore, the proof is based on the minimization of the $F$-radius and the $F_{W}$-radius of the zonotope that is computed by the observer. The $F$-radius of the zonotope in (11) can be obtained as

$$
J=\left\|\hat{H}_{k}^{\mathrm{x}_{\mathrm{io}}}\right\|_{F}^{2}=\operatorname{tr}\left(\hat{H}_{k}^{\mathrm{x}_{\mathrm{io}}} \hat{H}_{k}^{\mathrm{x}_{\mathrm{io}}^{\top}}\right)=\operatorname{tr}\left(P_{k}\right)
$$

where $J$ denotes the Frobenius radius and $P$ is the covariance of the zonotope matrix $\hat{H}_{k}^{\mathrm{x}_{\mathrm{io}}}$. Likewise, the $F_{W}$-radius of the zonotope in (11) will lead to

$$
J_{W}=\left\|H_{k+1}\right\|_{F, W}^{2}=\operatorname{tr} \hat{H}_{k}^{\mathrm{x}_{\mathrm{io}}} \hat{H}_{k}^{\mathrm{x}_{\mathrm{io}}{ }^{\top}}=\operatorname{tr}\left(W P_{k}\right),
$$

where $J_{W}$ denotes weighted Frobenius radius and $W P_{k}$ is the weighted-function covariance of the zonotope matrix $\hat{H}_{k}^{\mathrm{x}_{\mathrm{io}}}$. Hence, the Frobenius radius in (14) can be written as

$$
J=\operatorname{tr}\left(\left[\left(A-L_{k}^{\mathrm{io}} C\right) \hat{H}_{k}^{\hat{\mathrm{p}}^{\mathrm{io}}}-L_{k}^{\mathrm{io}} E_{\eta} E_{\omega}\right]\left[\left(A-L_{k}^{\mathrm{io}} C\right) \hat{H}_{k}^{\hat{\mathrm{p}}^{\mathrm{i}}}-L_{k}^{\mathrm{io}} E_{\eta} E_{\omega}\right]^{\top}\right) .
$$

Then, by considering $Q_{\omega}=E_{\omega} E_{\omega}^{\top}$ and $Q_{\eta}=E_{\eta} E_{\eta}^{\top}, 14$ can be rewritten as

$$
J=\operatorname{tr}\left(\left(A-L_{k}^{\mathrm{io}} C\right) P_{k}\left(A-L_{k}^{\mathrm{io}} C\right)^{\top}+L_{k}^{\mathrm{io}} Q_{\eta} L_{k}^{\mathrm{io}^{\top}}+Q_{\omega}\right) .
$$

Similarly, the weighted Frobenius radius $J_{w}$ in 15 is computed as

$$
J_{w}=\operatorname{tr}\left(W\left(A-L_{k}^{\mathrm{io}} C\right) P_{k}\left(A-L_{k}^{\mathrm{io}} C\right)^{\top}+W L_{k}^{\mathrm{io}} Q_{\eta} L_{k}^{\mathrm{io}^{\top}}+W Q_{\omega}\right)
$$

Therefore, the minimum $L^{\text {io }}$ is obtained when $\frac{\partial J_{w}}{\partial L^{\text {io }}}=0$, then using the definition of matrix trace in Appendix yields

$$
\partial_{L^{\mathrm{io}}} \operatorname{tr}\left(W L_{k}^{\mathrm{io}}\left(C P_{k} C^{\top}+Q_{\eta}\right)\right)=2 \partial_{L_{k}^{\mathrm{io}}} \operatorname{tr}\left(W A L_{k}^{\mathrm{io}} L_{k}^{\mathrm{io}{ }^{\top}}\right)
$$

where $\partial_{L^{\mathrm{i}}} \operatorname{tr}(\cdot)$ is a short notation for $\frac{\partial \operatorname{tr}(\cdot)}{\partial L^{\mathrm{io}}}$. If $W$ is an SPD matrix (see Appendix) and $W=W^{T}>0$, by transposition and left multiplication of $W^{-1}$ and considering Definition A6, it can be written that $-A P_{k} C^{\top}+L_{k}^{\mathrm{io}}\left(C P_{k} C^{\top}+Q_{\eta}\right)=0$. Then, the optimal gain $L_{k}^{\mathrm{io}, *}$ will be computed as it is written in 13,3

Note that the optimal observer gain in the case of using IOA (11) for the state estimation is computed by using Theorem 2

${ }^{3}$ The proof follows from the results available in 37 


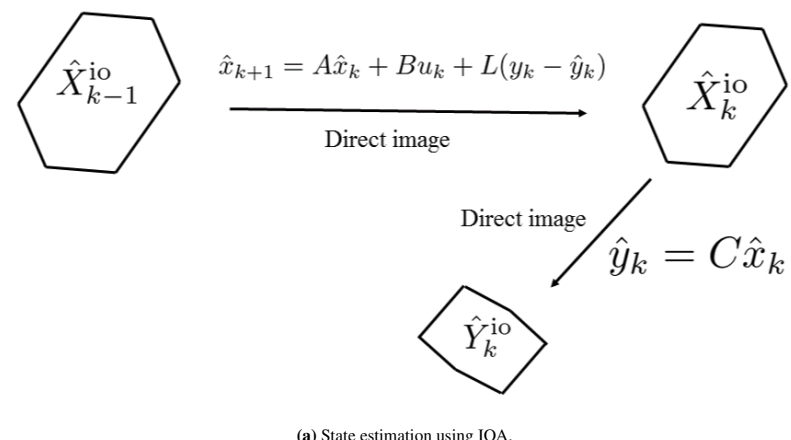

(a) State estimation using IOA.

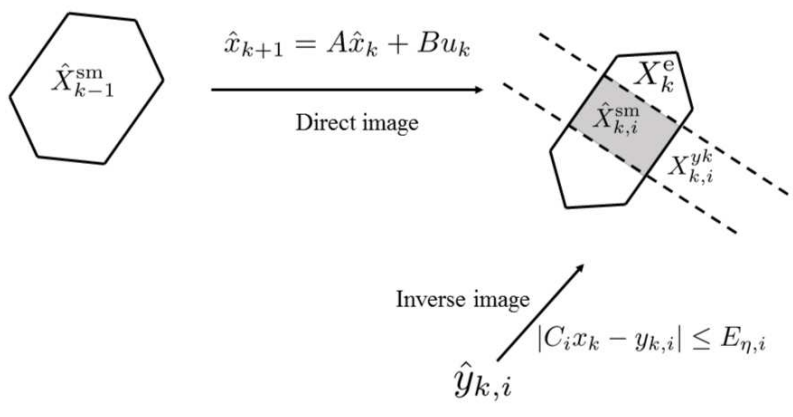

(b) State estimation using SMA.

FIGURE 1 Graphical interpretation of state estimation algorithms using IOA and SMA.

\section{4 | Comparison from the state estimation point of view}

The state estimation using SMA and IOA is introduced in Sections 2.2 and 2.3 based on ${ }^{10}$ and ${ }^{37}$, respectively. The main goal of this section is to compare these approaches from the state estimation point of view. Just for sake of clarity, the analytical comparison will be focused on the single-measurement case in this section as in $\frac{10}{10}$. But, according to $\frac{36}{6}$, the generalization of the comparison can be easily extended to the multi-measurement case considering Remark 2. It is worth mentioning that there are other manners to formulate the state estimation for the multi-measurement case using SMA approach based on ${ }^{36}$. But, the comparison will be less clear for the reader considering this approach.

The main difference between the SMA and IOA is related to the different way that measurements are taken into account to obtain the state estimation. This difference can be seen in Figure 1 , where the graphical interpretation of both approaches is presented. In the case of IOA, the state estimation is obtained in an explicit way by combining the model and the measurements through the observer gain. On the other hand, the SMA carries out this process implicitly by means of the intersection between the set of states consistent with the model and the measurements.

Comparing (5) with (12), it is not evident how to relate both state estimation approaches because of the different temporal information of the measured output used for each approach. More precisely, the state estimation using the IOA considers information of the output measurements at the previous time instant while state estimation using SMA is obtained by using the information of the measurements at the current time instant. But, in the case that the parameter $\lambda$ in SMA and the observer gain in IOA are identical, the same width of the state-bounding zonotopes will be obtained. However, the center of the state-bounding zonotopes are still different due to use of different temporal information of the measured output. Therefore, both state estimation approaches should be synchronized such that both use the measured output $y$ given at the same time instant.

Following ${ }^{38}$, the IOA proposed by ${ }^{37}$ can be modified leading to a current IOA (CIOA) given by

$$
\hat{\mathcal{X}}_{k}^{\text {cio }}=\hat{x}_{k}^{\mathrm{c}_{\text {cio }}} \oplus \hat{H}_{k}^{\mathrm{x}_{\text {cio }}} \mathbf{B}^{\mathrm{m}_{\mathrm{x}}+\mathrm{m}_{\omega}+1},
$$

where $\hat{x}_{k}^{\mathrm{c}_{\mathrm{cio}}}$ and $\hat{H}_{k}^{\mathrm{x}_{\mathrm{cio}}}$ denote the center and the segments of the zonotope (16) bounding the set of estimated states. Both center and segments can be propagated along the discrete-time instant $k$ as

$$
\begin{aligned}
\hat{x}_{k}^{\mathrm{c}_{\text {cio }}} & =\hat{p}_{k}^{\mathrm{c}_{\text {cio }}}+L_{k}^{\mathrm{cio}}\left(y_{k}-C \hat{p}_{k}^{\mathrm{c}_{\text {cio }}}\right), \\
\hat{H}_{k}^{\mathrm{x}_{\mathrm{cio}}} & =\left[\left(I-L_{k}^{\text {cio }} C\right) \hat{H}_{k}^{\hat{\mathrm{p}}^{\mathrm{cio}}}-L_{k}^{\text {cio }} E_{\eta}\right],
\end{aligned}
$$

with

$$
\begin{aligned}
\hat{p}_{k}^{\mathrm{c}_{\mathrm{cio}}} & =A \hat{x}_{k-1}^{\mathrm{c}_{\mathrm{cio}}}+B u_{k-1}, \\
\hat{H}_{k}^{\hat{\mathrm{p}}^{\mathrm{cio}}} & =\left[\begin{array}{ll}
A \hat{H}_{k-1}^{\mathrm{x}_{\mathrm{cio}}} & E_{\omega}
\end{array}\right] .
\end{aligned}
$$

As it can be seen from (16) and (17), the state estimation can be performed based on the information of the measurement given at the current time instant. When using the CIOA, the similarity of the $\hat{p}_{k}^{\mathrm{c}_{\mathrm{cio}}}$ with the $\hat{x}_{k}^{\mathrm{c}_{\mathrm{e}}}$ and also $\hat{H}_{k}^{\mathrm{p}^{\text {cio }}}$ with $H_{k}^{\mathrm{x}_{\mathrm{e}}}$ can be observed by comparing (17) with (5). It can also be noted that, using the same initial condition for both approaches, i.e., $x_{0}$ belongs to the both initial zonotope $\hat{\mathcal{X}}_{0}^{\text {cio }}=\hat{x}_{0}^{\mathrm{c}_{\text {cio }}} \oplus \hat{H}_{0}^{\mathrm{x}_{\text {cio }}} \mathbf{B}^{\mathrm{n}_{\mathrm{x}} \text { cio }}$ and $\hat{\mathcal{X}}_{0}^{\mathrm{sm}}=\hat{x}_{0}^{\mathrm{c}_{\mathrm{sm}}} \oplus \hat{H}_{0}^{\mathrm{x}_{\mathrm{sm}}} \mathbf{B}^{\mathrm{n}_{\mathrm{xsm}}}$, and taking into account the formulation of 
CIOA, the only difference between the approaches is related to the different manners of selecting the observer gain $L^{\text {cio }}$ and the parameter $\lambda$.

According to 10 , state estimation using SMA is parametrized by means of a vector $\lambda$ at each time instant $k$ in (4) and the proposed method to minimize the size of the zonotope (4) is designed based on the minimization of its segments. In addition, the optimal value of $\lambda$ is obtained as in $(9)$ according to $\frac{10}{10}$. Therefore, if the observer gain is designed following Theorem 3 the state-bounding zonotopes provided by CIOA and SMA are identical.

Theorem 3. Considering the dynamical model (1) and $x \in \hat{\mathcal{X}}^{\text {cio }}$ at time $k$, the optimal observer gain $L_{k}^{\text {cio,* }}$ that minimizes the F-radius of the state-bounding zonotope in (17) at time instant $k+1$ is computed as

$$
L_{k}^{\text {cio,* }}=\left(\hat{H}_{k}^{\mathrm{x}_{\text {cio }}} \hat{H}_{k}^{\mathrm{x}_{\mathrm{cio}}{ }^{\top}} C^{\top}\right)\left(C \hat{H}_{k}^{\mathrm{x}_{\text {cio }}} \hat{H}_{k}^{\mathrm{x}_{\text {cio }}^{\top}} C^{\top}+E_{\eta} E_{\eta}^{\top}\right)^{-1}
$$

Proof. Taking into account the segments of the zonotope $\hat{\mathcal{X}}_{k}^{\text {cio }}$ are obtained as the columns of the matrix in $(17 \mathrm{~b})$, the size of the estimated state at each time instant depends on the Frobenius norm of this matrix. Therefore, the optimal observer gain $L^{\text {cio }}$ given by $(18)$ is computed by minimizing the Frobenius norm of $\hat{H}_{k}^{\mathrm{x}_{\text {cio }}}$ in 17$)$. Furthermore, taking into account the facts that minimizing $F$-radius of a zonotope (which plays the same role as P-radius in LMI-based robust stability analysis) is equivalent to minimize the trace of its covariance and considering Definition A6 in Appendix, it can be written that

$$
\begin{aligned}
& \left\|\hat{H}_{k}^{\mathrm{x}_{\text {cio }}}\right\|_{\mathrm{F}}^{2}=\operatorname{tr}\left(\hat{H}_{k}^{\mathrm{x}_{\mathrm{cio}}}\left(\hat{H}_{k}^{\mathrm{x}_{\mathrm{cio}}}\right)^{\top}\right)=\operatorname{tr}\left(\left[\begin{array}{c}
\left(\Pi_{k} C\right) \hat{H}_{k}^{\hat{\mathrm{p}}^{\text {cio }}} \\
-L_{k}^{\text {cio }} E_{\eta}
\end{array}\right]\left[\left(\Pi_{k} C\right) \hat{H}_{k}^{\hat{\mathrm{p}}^{\text {cio }}}-L_{k}^{\text {cio }} E_{\eta}\right]\right) \\
& =\operatorname{tr}\left(\left[\begin{array}{cc}
\left(\left(\Pi_{k} C\right) \hat{H}_{k}^{\hat{\mathrm{p}}^{\mathrm{cio}}}\right)\left(\left(\Pi_{k} C\right) \hat{H}_{k}^{\hat{\mathrm{p}}^{\text {cio }}}\right)^{\top} & \left(\left(\Pi_{k} C\right) \hat{H}_{k}^{\hat{\mathrm{p}}^{\text {cio }}}\right)\left(-L_{k}^{\text {cio }} E_{\eta}\right)^{\top} \\
\left(-L_{k}^{\text {cio }} E_{\eta}\right)\left(\left(\Pi_{k} C\right) \hat{H}_{k}^{\hat{\mathrm{p}}^{\text {cio }}}\right)^{\top} & \left(-L_{k}^{\text {cio }} E_{\eta}\right)\left(-L_{k}^{\text {cio }} E_{\eta}\right)^{\top}
\end{array}\right]\right) \\
& =\left(\left(\Pi_{k} C\right) \hat{H}_{k}^{\hat{\mathrm{p}}^{\mathrm{cio}}}\right)\left(\left(\Pi_{k} C\right) \hat{H}_{k}^{\hat{\mathrm{p}}^{\text {cio }}}\right)^{\top}+\left(-L_{k}^{\text {cio }} E_{\eta}\right)\left(-L_{k}^{\text {cio }} E_{\eta}\right)^{\top} \text {, }
\end{aligned}
$$

where $\Pi_{k}=I-L_{k}^{\text {cio }}$. Therefore, the optimal value of the observer gain $L^{\text {cio }}$ is determined such that $\frac{\partial\left\|\hat{H}_{k}^{\mathrm{x}_{\mathrm{cio}}}\right\|_{\mathrm{F}}^{2}}{\partial L_{k}^{\text {cio }}}=0$. Thus, by considering [19], it can be written that

$$
-2 \hat{H}_{k}^{\hat{\mathrm{p}}^{\mathrm{cio}}} \hat{H}_{k}^{\hat{\mathrm{p}}^{\mathrm{cio}}{ }^{\top}}+2 L_{k}^{\mathrm{cio}, *} C \hat{H}_{k}^{\hat{\mathrm{p}}^{\mathrm{cio}}} \hat{H}_{k}^{\hat{\mathrm{p}}^{\mathrm{cio}}} C^{\top}+2 L_{k}^{\mathrm{cio}, *} E_{\eta} E_{\eta}^{\top}=0
$$

Finally, after the suitable manipulation of (20), the optimal observer gain can be expressed as in (18).

Therefore, in the case of IOA, the effect of measurements is considered through the selection of the observer gain. Thus, if the observer gain $L^{\text {io }}$ is selected as proposed in (13) according to the standard IOA, the value of the observer gain will depend on the relative values of the measurement noise and input disturbances as in the SMA. It means, the gain in the IOA and $\lambda$ in SMA play the same role for state estimation.

On the other hand, by designing the gain of the IOA as proposed by 37 , the formulation of the observer gain and the optimal parameter $\lambda^{*}$ proposed by ${ }^{10}$ for the SMA, are identical because these parameters are independent with respect to the information of the output measurements. Thus, for the temporal synchronization of both approaches, instead of using the standard structure of the IOA, the CIOA is used. Later on, CIOA is proposed based on using the zonotopic definition of the set for modeling the bounded uncertainties. Consequently, the optimal gain of CIOA is obtained based on minimizing the size of the obtained zonotope in (17). Therefore, the similarity of the SMA and the IOA is proved by introducing the CIOA.

\section{3 | FAULT DETECTION}

Model-based FD is based on comparing the measured outputs from the system with their estimation using the model of the healthy system. If an inconsistency is detected, the existence of a fault will be proved. So far, in this paper, IOA and SMA are both described and compared in the state estimation context. In this section, the comparison of both methods will be performed when applied to FD framework. In addition, the considered faults can be classified into different categories depending on its location as

- actuator faults, which affects the system inputs,

- sensor faults that affect the measurements of the inputs and outputs of the system. 


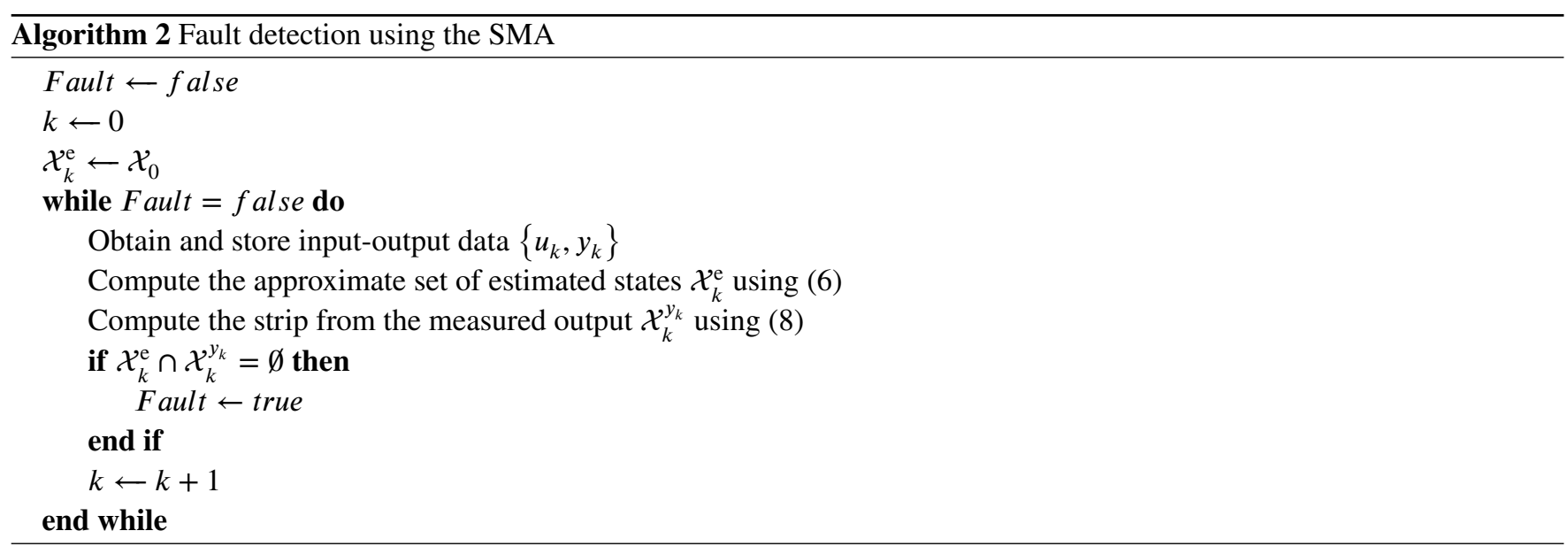

Furthermore, the model in (1) for the dynamical system including faults can be rewritten as

$$
\begin{aligned}
x_{k+1} & =A x_{k}+B u_{k}+E_{\omega} \omega_{k}+F^{a} f_{k}^{a}, \\
y_{k} & =C x_{k}+E_{\eta} \eta_{k}+F^{y} f_{k}^{y},
\end{aligned}
$$

where $f^{y}$ and $f^{a}$ represent output sensor and actuator faults, respectively, with their associated fault distribution matrices $F^{y} \in$ $\mathbb{R}^{n_{y} \times n_{y}}$ and $F^{a} \in \mathbb{R}^{n_{x} \times n_{y}}$. Furthermore, the relationship between the measured input $u_{k}^{*}$ and the real system input $u_{k}$ including the effect of faults at input sensors can be expressed as

$$
u_{k}^{*}=u_{k}+F^{u} f_{k}^{u}
$$

where $f^{u}$ indicates the input sensor fault with its associated matrix $F^{u} \in \mathbb{R}^{n_{u} \times n_{u}}$. In the case of an input sensor fault, the input of the resulting interval observer is assumed to be affected by the fault.

The sensitivity of the residual to a fault characterizes how the different considered actuator and sensor faults affect the residual. Mathematically speaking, it can be obtained as the difference between the residuals that are obtained from healthy and faulty operations of the system presented in (1) and (21), respectively, normalized by the fault $\operatorname{size}^{2}$.

Remark 3. In the minimum detectable fault analysis presented later in the paper, only one fault is considered at the same time. Therefore, those elements of $F$ and $f$ that are related to the considered fault should be considered while the other terms are neglected, e.g., in the case of actuator fault, $F^{a}$ and $f^{a}$ are considered and $F^{y}, f^{y}, F^{u}$ and $f^{u}$ are removed.

\section{1 | FD algorithm using SMA}

In general, the FD test using SMA is based on checking the intersection between the obtained zonotope in Step I and the strip in Step II of Algorithm 1 In the case that the intersection is empty, the existence of the fault will be proved, i.e., if

$$
\mathcal{X}_{k}^{\mathrm{e}} \cap \mathcal{X}_{k}^{y_{k}}=\emptyset
$$

the fault will be detected. Otherwise, the system is considered working in an healthy operation. Following the FD test based on the SMA, Algorithm 2 can be used.

Furthermore, the graphical interpretation of the proposed FD test in healthy and faulty cases is shown in Figure 2

As it can be seen from Figure $2 \mathcal{X}_{k}^{\mathrm{e}}$ and $\mathcal{X}_{k}^{y_{k}}$ are affected by the fault. Then, their intersection is empty and then the fault can be detected using Algorithm 2

As shown in Section 2.2 $\mathcal{X}_{k}^{\mathrm{e}}$ in Step I of Algorithm 1 can be computed as in (7). Then, given the zonotope $\mathcal{X}_{k}^{\mathrm{e}}$ in (7) and considering the vector $C_{i}$ from each measurement (where index $i$ indicates the $i^{\text {th }}$ row), the zonotope support strip $Q_{k}^{z}$ can be introduced at time instant $k$ as

$$
Q_{k}^{\mathrm{z}}=x_{k}: q_{k, i}^{\mathrm{z}_{\min }} \leq C_{i} x_{k} \leq q_{k, i}^{z_{\max }},
$$




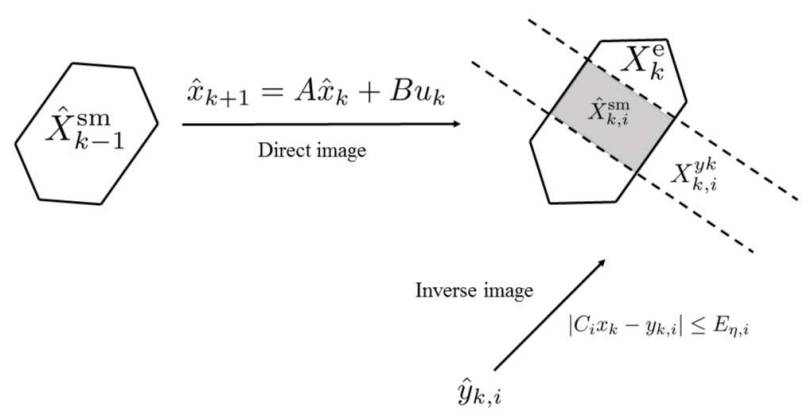

(a) Healthy operation of the system.

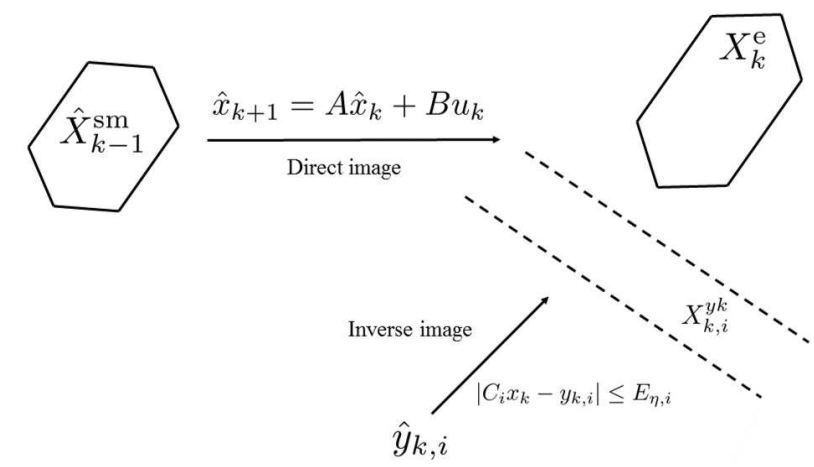

(b) Faulty operation of the system.

FIGURE 2 Graphical interpretation of FD test using the SMA.

where $q^{z_{\min }}$ and $q^{z_{\max }}$ are the minimum and the maximum values of the zonotope support strip, respectively, which can be computed as

$$
\begin{aligned}
& q_{k, i}^{z_{\min }}=C_{i} \hat{x}_{\mathrm{k}}^{\mathrm{c}_{\mathrm{e}}}-\left\|H_{\mathrm{k}}^{\mathrm{x}_{\mathrm{e}}^{\top}} C_{i}^{\top}\right\|_{1}, \\
& q_{k, i}^{z_{\max }}=C_{i} \hat{x}_{\mathrm{k}}^{\mathrm{c}_{\mathrm{e}}}+\left\|H_{\mathrm{k}}^{\mathrm{x}_{\mathrm{e}} \top} C_{i}^{\top}\right\|_{1} .
\end{aligned}
$$

As can be seen in (25), the effect of state disturbance is embedded in matrix $H^{\mathrm{x}_{\mathrm{e}}}$ that can be computed using (6b). Similarly, in order to consider the effect of measurement noise from the Step II of Algorithm 1 , the strip $Q^{\mathrm{s}}$ can be derived as

$$
Q_{k}^{\mathrm{s}}=\left\{x_{k} \in \mathbb{R}^{n_{x}}:\left|y_{k, i}-C_{i} x_{k}\right| \leq E_{\eta, i}\right\},
$$

where $y_{k, i}$ indicates each output measurement. The normalized form of the strip $Q_{s}$ can be derived as

$$
Q_{k}^{\mathrm{s}}=\left\{x_{k} \in \mathbb{R}^{n_{x}}:-1 \leq \frac{y_{k, i}}{E_{\eta, i}}-\frac{C_{i}}{E_{\eta, i}} x_{k} \leq+1\right\} .
$$

In general, each measurement $y$ allows to determine a set of consistent states as it is defined in (26), where $Q^{\mathrm{s}}$ is the region between two hyperplanes that is normalized in (27). Then, based on ${ }^{39}$, the computation of measurement strip can be done using (27) that is considered component-wise.

Thus, $q^{s_{\max }}$ and $q^{s_{\min }}$ values of the strip can be calculated at each time instant $k$ by using the measurement data in $(8)$ as

$$
\begin{aligned}
q_{k, i}^{\mathrm{s}_{\min }} & =\frac{y_{k, i}}{E_{\eta, i}}-1, \\
q_{k, i}^{\mathrm{s}_{\max }} & =\frac{y_{k, i}}{E_{\eta, i}}+1 .
\end{aligned}
$$

Then, the intersection between the zonotope support strip in 25] and the measurement strip in 28) is empty if

$$
\begin{aligned}
& q_{k, i}^{\mathrm{z}_{\min }}>q_{k, i}^{\mathrm{s}_{\max }}, \\
& q_{k, i}^{\mathrm{z}_{\max }}<q_{k, i}^{\mathrm{s}_{\min }} .
\end{aligned}
$$

Consequently, the fault will be detected if one of the conditions in (29) is satisfied. Based on conditions (29), the minimum detectable abrupt fault will be characterized for the considered type of faults assuming that they are introduced as in 21 .

\section{Case I: Minimum detectable output sensor fault}

Considering the dynamical model (21) and Remark3 in the case of output sensor fault, the minimum magnitude of output sensor fault that can be detected is obtained by using 29$]$ and following Theorem 4.

Theorem 4. (Minimum detectable output sensor fault) Considering the faulty dynamical model (21) and the conditions (29), the minimum detectable abrupt output sensor fault can be characterized as

$$
\psi_{i}^{y} f_{k, i}^{y}>2\left\|H_{\mathrm{k}}^{\mathrm{x}_{\mathrm{e}}^{\top}} C_{i}^{\top}\right\|_{1}+2,
$$


where the term $\Psi^{y}=\frac{F^{y}}{E_{\eta}}$ represents the sensitivity with respect to the considered output sensor fault while the obtained expression for the output sensor fault is normalized using $E_{\eta}$. Moreover, the value 2 refers to the consideration of the worst-case scenario, i.e., the prediction is considered with the extreme value (it is located at either the lower or the upper bound of the considered threshold).

Proof. Following Algorithm 2, it can be written that

$$
C_{i} \hat{x}_{\mathrm{k}}^{\mathrm{c}_{\mathrm{e}}}+\left\|H_{\mathrm{k}}^{\mathrm{x}_{\mathrm{e}}^{\top}} C_{i}^{\top}\right\|_{1}<\frac{y_{k, i}}{E_{\eta, i}}-1 .
$$

Then, considering the dynamical model (21), 31] becomes

$$
C_{i} \hat{x}_{\mathrm{k}}^{\mathrm{c}_{\mathrm{e}}}+\left\|H_{\mathrm{k}}^{\mathrm{x}_{\mathrm{e}}^{\top}} C_{i}^{\top}\right\|_{1}<C_{i} x_{k}+\frac{\eta_{k_{i}}}{E_{\eta_{i}}}+\psi_{i}^{y} f_{k, i}^{y}-1 .
$$

Moreover, by considering the worst-case scenario, where the disturbances have a maximal influence in the opposite direction compared to that of the occurring fault, (32) can be written in faultless scenario, i.e., $f_{k}^{y}=0$, as

$$
C_{i} \hat{x}_{\mathrm{k}}^{\mathrm{c}_{\mathrm{e}}}+\left\|H_{\mathrm{k}}^{\mathrm{x}_{\mathrm{e}}^{\top}} C_{i}^{\top}\right\|_{1}=C_{i} x_{k}+\frac{\eta_{k_{i}}}{E_{\eta_{i}}}-1
$$

Then, considering the worst-case scenario, (31) can be written as $C_{i} \hat{x}_{\mathrm{k}}^{\mathrm{c}_{\mathrm{e}}}+\left\|H_{\mathrm{k}}^{\mathrm{x}_{\mathrm{e}}^{\top}} C_{i}^{\top}\right\|_{1}=2\left\|H_{\mathrm{k}}^{\mathrm{x}_{\mathrm{e}}^{\top}} C_{i}^{\top}\right\|_{1}$. It means, $x_{k}$ is placed in the border of the zonotope support strip (the measurement strip is placed in the farthest possible location). Also, minimizing $C_{i} x_{k}+\frac{\eta_{k_{i}}}{E_{\eta_{i}}}$, the minimum detectable abrupt output sensor fault can be expressed as in 30] using (33).

\section{Case II: Minimum detectable input sensor fault}

The minimum detectable input sensor fault can be computed based on the dynamical model (21) and the faulty input (22) that is injected to the model. Therefore, (21) can be rewritten as in (1). But, in this case, the model/observer is affected by the faulty input (22). In this regard, (6) is expressed as

$$
\begin{aligned}
\hat{x}_{k}^{\mathrm{c}_{\mathrm{e}}} & =A \hat{x}_{k-1}^{\mathrm{c}}+B\left(u_{k-1}+F^{u} f_{k-1}^{u}\right), \\
H_{k}^{\mathrm{x}_{\mathrm{e}}} & =\left[\begin{array}{ll}
A \hat{H}_{k-1}^{\mathrm{x}} & E_{\omega}
\end{array}\right] .
\end{aligned}
$$

Now, the FD test is based on checking the intersection between the zonotope support strip and the measurement strip. Moreover, the minimum magnitude of the abrupt input sensor fault that can be detected can be determined by means of Theorem 5

Theorem 5. (Minimum detectable input sensor fault) Considering (34) and the conditions (29), the minimum detectable abrupt input sensor fault can be expressed as

$$
\psi_{i}^{u} f_{k, i}^{u}>2\left\|H_{\mathrm{k}}^{\mathrm{x}_{\mathrm{e}}^{\top}} C_{i}^{\top}\right\|_{1}+2,
$$

where the term $\Psi^{u}=-C B F^{u}$ is the sensitivity with respect to the considered input sensor fault.

Proof. Considering that Algorithm 2 can be used for detecting the fault based on SMA, the FD test is performed by checking the intersection between the zonotope support strip and the measurement strip that can be computed in the same way as in 25. and (28), respectively. Thus, in the case of input sensor fault, the conditions (29) can be rewritten as

$$
C_{i} \hat{x}_{\mathrm{k}}^{\mathrm{c}_{\mathrm{e}}}+\psi_{i}^{u} f_{k, i}^{u}+\left\|H_{\mathrm{k}}^{\mathrm{x}_{\mathrm{e}}^{\top}} C_{i}^{\top}\right\|_{1}<\frac{y_{k, i}}{E_{\eta, i}}-1 .
$$

Hence, by assuming the worst-case scenario, the expression (35) can be obtained by the suitable manipulation of (36).

It is worth to mention that the detection of the input sensor fault depends on the direction of vector $C_{i}$ and the input of the system. Thus, the input should be designed to guarantee that the input sensor fault is not in the orthogonal direction of vector $C_{i}$. Otherwise, the fault can not be detected. 


\section{Case III: Minimum detectable actuator fault}

Considering the dynamical model 21] and Remark 3 in the case of actuator fault, the minimum magnitude of abrupt actuator fault that can be detected is obtained by using 29 , and following Theorem 6 .

Theorem 6. (Minimum detectable actuator fault) Considering the dynamical model (21) and the conditions (29), the minimum detectable abrupt actuator fault can be computed as

$$
\psi_{i}^{a} f_{k, i}^{a}>2\left\|H_{k}^{\mathrm{x}_{\mathrm{e}}^{\top}} C_{i}^{\top}\right\|_{1}+2,
$$

where $\Psi^{a}=C F^{a}$ is the sensitivity with respect to the given actuator fault.

Proof. Considering the faulty dynamical model 21 and conditions 29 to guarantee the detection of the fault, it can be written that

$$
C_{i} \hat{x}_{k}^{\mathrm{c}_{\mathrm{e}}}+\left\|H_{k}^{\mathrm{x}_{\mathrm{e}}^{\top}} C_{i}^{\top}\right\|_{1}<C_{i} x_{k}+\frac{\eta_{k, i}}{E_{\eta, i}}+\psi_{i}^{a} f_{k, i}^{a}-1
$$

Therefore, the expression of the minimum detectable actuator fault can be obtained as it is written in (37) by considering the worst-case scenario and adequate manipulation of 38 .

Similarly to the case of input sensor fault, the minimum magnitude of the actuator fault that can be detected is related to the direction of the vector $C_{i}$ and the input. Therefore, the proper input should be designed to avoid the problem of having the actuator fault and vector $C_{i}$ in the same orthogonal direction.

Remark 4. It can be noted that the interpretation of the term $2\left\|H_{k}^{\mathrm{X}_{\mathrm{e}} \top}\right\|_{1}$ in the case of SMA stands for the width of the zonotopes $\mathcal{X}^{\mathrm{e}}$ in (7), where the effect of state disturbance is taken into account. On the other hand, the effect of measurement noise is considered through $Q^{s}$ in 27. Then, based on the consideration of the two mentioned uncertainties, it can be guaranteed that a fault with higher effect than the effect of uncertainties is detectable. Therefore, the characterized minimum detectable fault is known as the smallest magnitude of the considered fault whose effect is higher than the effect of the uncertainties.

\section{2 | FD algorithm using the IOA}

Now, the IOA is applied to FD in order to characterize the minimum detectable fault for the same type of considered faults than in the case of SMA. The standard procedure of the FD test based on IOA relies on checking the inconsistency of the measurements with their provided estimations by the observer model. In other words, any inconsistency between the measured outputs using sensors and the estimated behavior that is computed by using the system model, called residual, is known as a fault occurrence $4^{4}$ However, considering the standard method of residual generation in order to characterize the minimum detectable fault, it is not possible to determine a clear relationship of IOA and SMA in FD framework since a common FD test that allows relating both approaches is required. A possible solution in order to homogenize the approaches is to use the zonotope support strip in the case of IOA for formulating the FD conditions. In this context, the generated residual is formulated in terms of strip mathematical expression that can be compared with the SMA. Moreover, the strip $\mathcal{X}_{k}^{y_{k}}$ can be obtained using [27], where the effect of the measurement noise is taken into account (same as the Step II in the case of SMA). On the other hand, considering the vector $C_{i}$, the zonotopic support strip $Q^{x}$ can be defined based on using the state estimation of the IOA in (12) at each time instant $k$ as

$$
Q_{k}^{x}=\left\{x_{k}: q_{k, i}^{\mathrm{x}_{\min }} \leq C_{i} x_{k} \leq q_{k, i}^{\mathrm{x}_{\max }}\right\}
$$

where $q_{k}^{\mathrm{x}_{\min }}$ and $q_{k}^{\mathrm{x}_{\max }}$ are the minimum and the maximum values of the zonotope support strip, respectively. Furthermore, $q^{\mathrm{x}_{\min }}$ and $q^{\mathrm{x}_{\max }}$ are determined at each time instant $k$ as

$$
\begin{aligned}
& q_{k, i}^{\mathrm{x}_{\min }}=C_{i} \hat{x}_{k}^{\mathrm{c}_{\mathrm{io}}}-\left\|H_{k}^{\mathrm{x}_{\mathrm{io}} \top} C_{i}^{\top}\right\|_{1}, \\
& q_{k, i}^{\mathrm{x}_{\max }}=C_{i} \hat{x}_{k}^{\mathrm{c}_{\mathrm{io}}}+\left\|H_{k}^{\mathrm{x}_{\mathrm{io}} \top} C_{i}^{\top}\right\|_{1},
\end{aligned}
$$

where the effect of the state disturbance is embedded into matrix $H^{\mathrm{x}_{\mathrm{io}}}$, which can be computed using $12 \mathrm{~b}$.

Therefore, the existence of the fault will be proved by checking

$$
\hat{\mathcal{X}}_{k}^{\mathrm{io}} \cap \mathcal{X}_{k}^{y_{k}}=\emptyset
$$

${ }^{4} \mathrm{See}{ }^{2}$ for further details. 


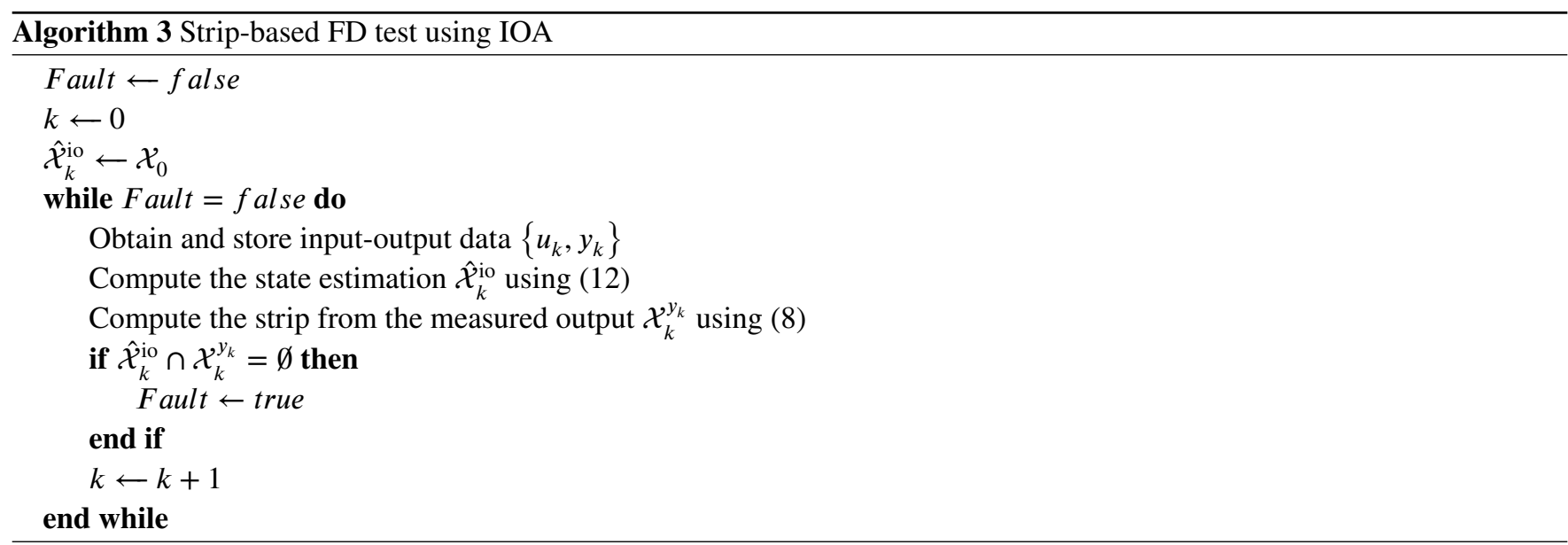

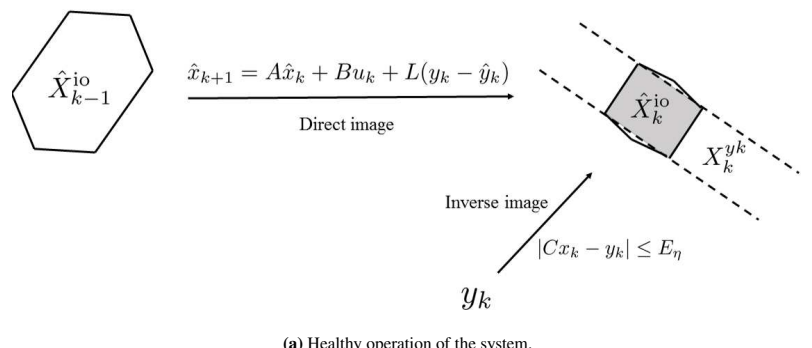

(a) Healthy operation of the system.

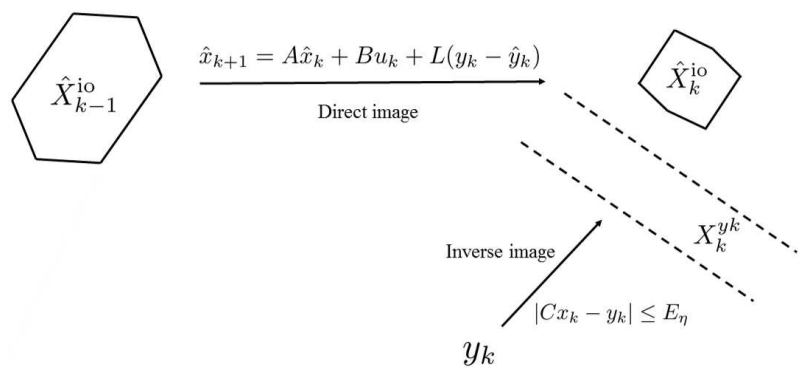

(b) Faulty operation of the system.

FIGURE 3 Graphical interpretation of the strip-based FD test using IOA.

such that in the case of empty intersection, the fault will be detected. Otherwise, FD cannot be guaranteed. Thus, one of the following conditions should be satisfied in the case of faulty operation of the system:

$$
\begin{aligned}
& q_{k, i}^{\mathrm{x}_{\min }}>q_{k, i}^{\mathrm{s}_{\max }}, \\
& q_{k, i}^{\mathrm{x}_{\max }}<q_{k, i}^{\mathrm{s}_{\min }} .
\end{aligned}
$$

Algorithm 3 summarizes the strip-based FD test using IOA. Moreover, the graphical interpretation of Algorithm 3 is shown in Figure 3 .

Next, the FD performance for different type of abrupt faults will be characterized when the FD test in (41) is considered.

Remark 5. Note that the interpretation of the term $2\left\|H_{k}^{\mathrm{x}_{\mathrm{i}}{ }^{\top}} C_{i}^{\top}\right\|_{1}$ stands for the width of the zonotopes $\mathcal{X}^{\text {io }}$ in (11), where the effect of state disturbance is taken into account. On the other hand, the effect of measurement noise is considered through $Q^{s}$ in (27). Then, based on the consideration of two mentioned uncertainties, it can be guaranteed that the fault with higher effect in comparison with the effect of uncertainties can be detected.

\section{Case I: Minimum detectable output sensor fault}

The system to be monitored in the case of output sensor fault can be described as (21). Considering the zonotope support strip in (40) and the normalized form of the measurements strip in (28), the minimum detectable abrupt output sensor fault based on the IOA can be computed by using Theorem 7

Theorem 7. (Minimum detectable output sensor fault) Considering the faulty dynamical model (21) and the conditions (42), the minimum magnitude of the abrupt output sensor fault that is detectable in one step can be computed as

$$
\psi_{i}^{y} f_{k, i}^{y}>2\left\|H_{k}^{\mathrm{x}_{\mathrm{io}} \top} C_{i}^{\top}\right\|_{1}+2 .
$$


Proof. Considering the dynamical model (21), by substituting the zonotope support strip in (40) and the normalized form of the measurements strip in (28) into one of the faulty conditions (42) yields

$$
C_{i} \hat{x}_{k}^{\mathrm{c}_{\mathrm{io}}}-\left\|H_{k}^{\mathrm{x}_{\mathrm{io}}^{\top}} C_{i}^{\top}\right\|_{1}<C_{i} x_{k}+\frac{\eta_{k, i}}{E_{\eta_{i}}}+\psi_{i}^{y} f_{k, i}^{y}-1 .
$$

Moreover, considering the worst-case value of $x_{k}$ and minimizing $C_{i} x_{k}+\frac{\eta_{k, i}}{E_{\eta, i}}$, the minimum detectable output sensor fault can be characterized as in (43) after the algebraic manipulation of 444.

\section{Case II: Minimum detectable input sensor fault}

Considering the dynamical model (21) and substituting faulty input (22) in (12) for the input $u$ gives

$$
\begin{aligned}
\hat{x}_{k}^{\mathrm{c}_{\mathrm{io}}} & =\left(A \hat{x}_{k-1}^{\mathrm{c}_{\mathrm{io}}}+B\left(u_{k-1}+F^{u} f_{k-1}^{u}\right)+L_{k}^{\mathrm{io}}\left(y_{k-1}-C\left(A \hat{x}_{k-1}^{\mathrm{c}_{\mathrm{io}}}+B\left(u_{k-1}+F^{u} f_{k-1}^{u}\right)\right),\right.\right. \\
\hat{H}_{k}^{\mathrm{x}_{\mathrm{io}}} & =\left[\left(I-L_{k}^{\mathrm{io}} C\right) \hat{H}_{k}^{\hat{\mathrm{p}}^{\mathrm{io}}}-L_{k}^{\mathrm{io}} E_{\eta}\right] .
\end{aligned}
$$

Now, by considering [45, the minimum detectable abrupt input sensor fault can be calculated by the following Theorem 8 .

Theorem 8. (Minimum detectable input sensor fault) Considering the faulty model (45) and the conditions (42), the minimum detectable abrupt input sensor fault can be characterized as

$$
\psi_{i}^{u} f_{k}^{u}>2\left\|H_{\mathrm{k}}^{\mathrm{x}_{\mathrm{io}}^{\top}} C_{i}^{\top}\right\|_{1}+2
$$

Proof. Considering the faulty model (45), the faulty conditions (42) can be rewritten by using (40) and (28) as

$$
C_{i} \hat{x}_{\mathrm{k}}^{\mathrm{c}_{\mathrm{io}}}+\psi_{i}^{y} f_{k, i}^{u}+\left\|H_{\mathrm{k}}^{\mathrm{x}_{\mathrm{io}}^{\top}} C_{i}^{\top}\right\|_{1}<\frac{y_{k, i}}{E_{\eta, i}}-1 .
$$

Then, the minimum detectable input sensor fault can be characterized as 46 by assuming the worst-case scenario and the corresponding manipulation of 47].

Note from (46) that the magnitude of the minimum detectable input sensor fault depends on the input and the direction of the vector $C_{i}$. Therefore, the input $u$ should be designed in the way that the orthogonal direction of the vector $C_{i}$ and the direction of the given fault will be different. Otherwise, the input sensor fault will never be detected.

\section{Case III: Minimum detectable actuator fault}

Considering the faulty dynamical model 21 , the minimum detectable abrupt actuator fault can be computed following the Theorem 9

Theorem 9. (Minimum detectable actuator fault) Considering the faulty dynamical model (21) and the conditions $(42)$, the minimum detectable abrupt actuator fault can be calculated as

$$
\psi_{i}^{a} f_{k, i}^{a}>2\left\|H_{k}^{\mathrm{x}_{\mathrm{io}}^{\top}} C_{i}^{\top}\right\|_{1}+2 .
$$

Proof. Considering the dynamical model (21) and inserting the strips (40) and (28) into one of the faulty conditions (42) yields

$$
C_{i} \hat{x}_{k}^{\mathrm{c}_{\mathrm{io}}}-\left\|H_{k}^{\mathrm{x}_{\mathrm{io}}^{\top}} C_{i}^{\top}\right\|_{1}<C_{i} x_{k}+\frac{\eta_{k, i}}{E_{\eta, i}}+\psi_{i}^{a} f_{k, i}^{a}-1 .
$$

Consequently, the minimum magnitude of the actuator fault that can be detected is derived based on (49) by assuming the worst-case value of $x_{k}$ and $\eta_{k}$ as it is expressed in 48.

Additionally, the detection of the actuator fault is related to the orthogonal direction of the vector $C_{i}$. Thus, in order to guarantee the fault detection, the input $u$ should be designed in such a way that the orthogonal direction of the vector $C_{i}$ and the direction of the given fault will be different. 


\section{3 | Comparative assessment}

Algorithm 3 leads to obtain the comparable structure of the IOA and the SMA in FD framework. Consequently, the minimum detectable abrupt fault that is derived by the presented approaches can be compared with each other under this circumstance. Therefore, the key point of FD test is to check the intersection between the measurement strip and the state estimation support strip comparing Algorithm 2 with Algorithm 3

Actually, the formulation of the minimum detectable fault is characterized based on the conditions (29) and (42) considering Algorithms 2 and 3 for both the SMA and IOA, respectively. As it can be observed from Algorithms 2 and 3 the effect of the measurement noise is considered when determining the measurement strip (26) in both approaches. Furthermore, in the case of SMA, the effect of state disturbance is considered through the computation of zonotope support strip in (24), where the state disturbance effect is embedded in the matrix $H^{\mathrm{x}_{\mathrm{e}}}$ that can be obtained using 6b. Moreover, in the case of IOA, the effect of the state disturbance is taken into account similarly as the IOA through the zonotope support strip [39], where the state disturbance effect is embedded in the matrix $H^{\mathrm{x}^{\mathrm{io}}}$ that can be obtained using (12b). In both IOA and SMA, the strip from the measurement data can be obtained considering the unknown but bounded uncertainties as in 277, where the normalized form of the maximum and the minimum values of the output prediction can be defined as upper and lower bounds of the strip that is formulated in (28). On the other hand, the supporting hyperplanes are characterized using the obtained sets from state estimation. Then, according to Algorithm 2, the approximated set of the estimated states $\mathcal{X}_{k}^{\mathrm{e}}$ can be used for computing the zonotope support strip in (25). Meanwhile, according to Algorithm 3 the zonotope support strip that is computed in (40) is generated using the state-bounding zonotope $\hat{\mathcal{X}}_{k}^{\text {io }}$.

Furthermore, the minimum detectable sensor and actuator faults are characterized based on the SMA in (30), (35) and (37), respectively. Besides, the minimum magnitude of same kind of faults that can be detected are derived in (43), 446, and (48) based on IOA.

From the mathematical comparison of both approaches, it can be noted that in the SMA, the minimum detectable fault magnitude depends on the width of the zonotope $\mathcal{X}_{k}^{\mathrm{e}}$, which is obtained from the first step of its state estimation algorithm. On the other hand, the characterization of the minimum detectable fault based in the IOA depends on the width of the zonotope $\hat{\mathcal{X}}_{k}^{\text {io }}$, which is obtained from its state estimation. Indeed, the magnitude of the minimum detectable fault in SMA is related to the $H_{k}^{\mathrm{x}_{\mathrm{e}}}$ that is obtained based on (7) as $H_{k}^{\mathrm{x}_{\mathrm{e}}}=\left[A \hat{H}_{k-1}^{\mathrm{x}} E_{\omega}\right]$. Besides, the minimum magnitude of the fault that can be detected based on IOA depends on $\hat{H}_{k}^{\mathrm{x}_{\mathrm{io}}}$ that, according to $(12)$, it can be written as $\hat{H}_{k}^{\mathrm{x}_{\mathrm{io}}}=\left[\left(I-L_{k}^{\mathrm{io}} C\right) A \hat{H}_{k-1}^{\mathrm{x}_{\mathrm{io}}}\left(I-L_{k}^{\mathrm{io}} C\right) E_{\omega}-L_{k}^{\mathrm{io}} E_{\eta}\right]$.

Furthermore, the influence of the observer gain should be considered in computing the minimum detectable fault. It means, in the IOA, the observer gain $L^{\text {io }}$ is not only designed to guarantee the convergence of the observer, but also it can be used to guarantee the desired FD performance. From the mathematical point of view, when the observer gain is considered null $\left(L^{\text {io }}=0\right.$ ), the same formulation of the minimum detectable fault is obtained in both approaches since $\hat{H}^{\mathrm{x}_{\mathrm{io}}}=H^{\mathrm{x}_{\mathrm{e}}}$. But, it should be considered that in the case that $L^{\text {io }}=0$, the observer becomes a simulator and it can only be used in ideal conditions and with perfect knowledge of the initial states. Thus, in the case of $L^{\text {io }}=0$, the minimum detectable fault determined by the SMA and IOA are identical. Otherwise, the FD test using IOA is more conservative in general than the SMA one.

Moreover, as it was explained before, the difference between the IOA and SMA in the state-estimation framework is related to the use of different temporal information from the given measurement $y$. Therefore, by considering the same initial conditions, the same values of $\lambda^{*}$ and $L^{\mathrm{io}, *}$ can be obtained in steady state when using (9) and (13), respectively. Furthermore, if $\lambda^{*}=L^{\mathrm{i}, *}$ and when $k$ tends to infinity, it will be obtained that $\hat{H}_{\infty}^{\mathrm{x}_{\mathrm{io}}}=H_{\infty}^{\mathrm{x}_{\mathrm{e}}}$. Then, the only difference is related to the center of the statebounding zonotope. Therefore, the identical minimum detectable fault can be computed based on both IOA and SMA since the mathematical expression of the minimum magnitude of the fault that can be detected is independent of the center of the state-bounding zonotope.

Furthermore, the advantage of IOA is related to the observer gain that can be tuned to obtain better FD performance. Moreover, another advantage of IOA in comparison with SMA is related to fact that after the fault occurrence, it is not possible to monitor the system behaviour with the SMA. But, IOA can monitor the system even after the fault has appeared. This issue is a major limitation in the use of SMA in FD applications.

In Section 2.4, the approaches are synchronized by modifying IOA to the form of CIOA from state estimation point of view. Using the information of the measurements at current time instant based on the CIOA leads to obtain the same state estimation using IOA and SMA 5 So far, according to Algorithm 3 the strip can be obtained using $(8)$ and the state estimation is obtained using (17). When the current information of the output for state estimation is used, both (8) and (17) are influenced by the fault,

\footnotetext{
${ }^{5}$ see the formulation of the state estimation in $[17$
} 


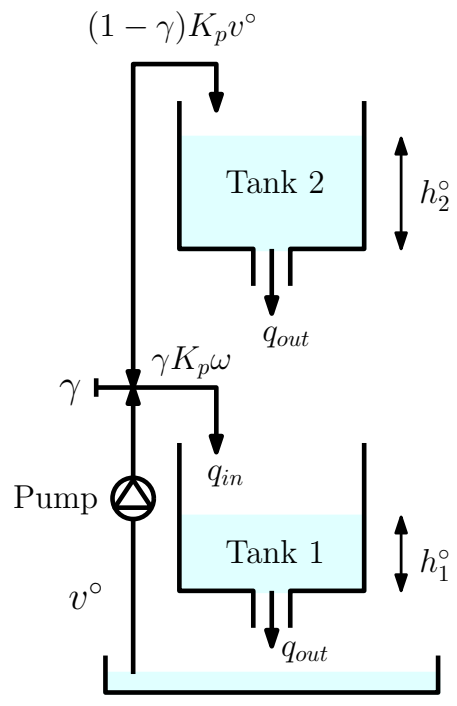

FIGURE 4 Schematic diagram of the two-tanks system.

simultaneously. Therefore, the empty intersection cannot be obtained since (8) and (17) are moved together by the influence of the fault. Therefore, the intersection will never be empty. Consequently, the fault will never be detected. Hence, the CIOA can not be used for FD.

\section{4 | CASE STUDY}

\section{1 | Plant Description}

The illustration of comparative analysis of IOA and SMA will be performed by using the two-tanks system, which is based on ${ }^{40}$. The scheme of the considered case study is shown in Figure 4

The input of the two-tanks system is the pump flow rate that is determined by the voltage of the pump $v$ and the state of the system is the water level of the tanks. Additionally, Tank 1 is being affected by the outflow of Tank 2 . Furthermore, the inflow of Tank 1 is also affected by an additional disturbance $\omega$ that is generated by the uncertain position of the valve $\gamma$, where that position can vary between 0 and 1 , i.e., $0 \leq \gamma \leq 1$. Moreover, both upper and lower tanks are made of Plexiglas tubes with the height of $20 \mathrm{~cm}$ and the connection of the tanks and the pump is done using a pipe with a diameter $6 \mathrm{~mm}$.

The mathematical model of the system is derived using mass-balance relations and the Bernoulli's law as follows:

$$
\begin{aligned}
& \frac{d h_{1, t}^{\circ}}{d t}=-\frac{a_{1}}{A_{1}} \sqrt{2 g h_{1, t}^{\circ}}+\frac{a_{2}}{A_{1}} \sqrt{2 g h_{2, t}^{\circ}}+\frac{\gamma K_{p}}{A_{1}} \omega_{t}, \\
& \frac{d h_{2, t}^{\circ}}{d t}=-\frac{a_{2}}{A_{2}} \sqrt{2 g h_{2, t}^{\circ}}+\frac{(1-\gamma) K_{p}}{A_{2}} v_{t}^{\circ},
\end{aligned}
$$

where

- $K_{p} v_{t}^{\circ}$ is the flow through the pump.

- $(1-\gamma) K_{p} v_{t}^{\circ}$ is the flow towards Tank 1 according to the valve position $\gamma$.

- $\gamma K_{p} \omega_{t}$ is the flow towards Tank 2 according to the valve position $\gamma$.

- $K_{p}$ is the pump constant.

- $v_{t}^{\circ}$ is the velocity of the water flow through the pump.

- $A_{i}$ is the cross section of Tank $i$, with $i=1,2$.

- $a_{i}$ is the cross sectional area of the outlet pipes, with $i=1,2$.

- $g$ is acceleration due to gravity.

- $h_{i}^{\circ}$ is the level of the water in Tank $i$, with $i=1,2$.

Furthermore, the non-linear model 50 ) is linearized around the following working point: 
- $h_{1}^{*}=12.4 \mathrm{~cm}$,

with the following parameter values:

- $K_{p}=3.35 \mathrm{~cm}^{3} / \mathrm{Vs}$,
- $h_{2}^{*}=1.8 \mathrm{~cm}$,
- $v^{*}=3.00 \mathrm{~V}$,

Hence, the linearized model of 50 around the working point that is presented using the superscript $*$ can be obtained by introducing the variables $h_{i}=h_{i}^{\circ}-h_{i}^{*}$ and $v_{i}=v_{i}^{\circ}-v_{i}^{*}$ as

$$
\begin{aligned}
& \dot{h}_{t}=\left[\begin{array}{cc}
-\frac{1}{T_{1}} & \frac{A_{2}}{A_{1} T_{2}} \\
0 & -\frac{1}{T_{2}}
\end{array}\right] h_{t}+\left[\begin{array}{cc}
\frac{\gamma K_{p}}{A_{1}} & 0 \\
0 & \frac{(1-\gamma) K_{p}}{A_{2}}
\end{array}\right] u_{t}, \\
& y_{t}=\left[\begin{array}{ll}
K_{c} & 0
\end{array}\right] h_{t},
\end{aligned}
$$

where $u_{t}=\left[\begin{array}{l}\omega \\ v\end{array}\right]$ and $K_{c}$ is a experimental estimated parameter. Moreover, $T_{i}=\frac{A_{i}}{a_{i}} \sqrt{\frac{2 h_{i}^{*}}{g}}$ with $i=1$, 2. According to ${ }^{40}$, the parameters of the model in 51, are given as $A_{1}=A_{2}=28 \mathrm{~cm}^{2}, a_{1}=a_{2}=0.071 \mathrm{~cm}^{2}, K_{c}=0.50 \mathrm{~V} / \mathrm{cm}, g=981 \mathrm{~cm} / \mathrm{s}^{2}$, and hence $T_{1}=62.7034 \mathrm{~s}$ and $T_{2}=23.8900 \mathrm{~s}$. Using the Euler discretization with a sampling time of $1 \mathrm{~s}$, the linearized model of this system can be written in the state-space form as

$$
\begin{aligned}
h_{k+1} & =A h_{k}+B v_{k}+E_{\omega} \omega_{k}, \\
y_{k} & =C h_{k}+E_{\eta} \eta_{k},
\end{aligned}
$$

where

$$
A=\left[\begin{array}{cr}
0.9842 & 0.0407 \\
0 & 0.9590
\end{array}\right], \quad B=\left[\begin{array}{l}
0.0007 \\
0.0352
\end{array}\right], \quad C=\left[\begin{array}{ll}
0.5 & 0
\end{array}\right] .
$$

Moreover, bounded disturbances influencing all the state-space directions and the measurement noise are modeled respectively with $E_{\omega}$ and $E_{\eta}$ as

$$
E_{\omega}=\left[\begin{array}{cc}
0.08 & 0 \\
0 & 0.08
\end{array}\right], \quad E_{\eta}=[0.01] .
$$

In [52, the output $y$ is the voltage obtained from the level sensor. Hence, considering the physical features of the twotank system, the range of the measured output is $[0,10] \mathrm{V}$ since the height of the each tank is from $0 \mathrm{~cm}$ up to $20 \mathrm{~cm}$ and $K_{c}=0.5 \mathrm{~V} / \mathrm{cm}$. Moreover, based on the experiment, the incremental value of the measured output around the working point of the lower tank is from $4 \mathrm{~V}$ up to $8 \mathrm{~V}$ (or $8 \mathrm{~cm}$ to $16 \mathrm{~cm}$ ).

\section{2 | State estimation}

In this section, the state estimation using IOA, CIOA and SMA is compared in order to validate the obtained mathematical analyses presented in Section 2

As it is derived in Section 2, the state estimation using SMA in (4) at time instant $k$ can be obtained by using Algorithm 1 On the other hand, the state estimation based on IOA at time instant $k$ can be computed using the following information of the output measurement:

- the information of the measured output at the previous time instant $y_{k-1}$ using (11) and (12),

- the information of the measured output at the current time instant $y_{k}$ using (16) and (17).

The state estimation using IOA, CIOA and SMA approaches at time instant $k=100$ are shown in Figure 5 As can be observed from Figure 5 , the state estimation using the IOA and CIOA are different because of the different temporal information of the measured output. But, when the CIOA is used instead of IOA, the identical state estimation as SMA will be obtained. Notice that the same initial conditions are considered for obtaining the results in Figure 5 During the simulation, the optimal 


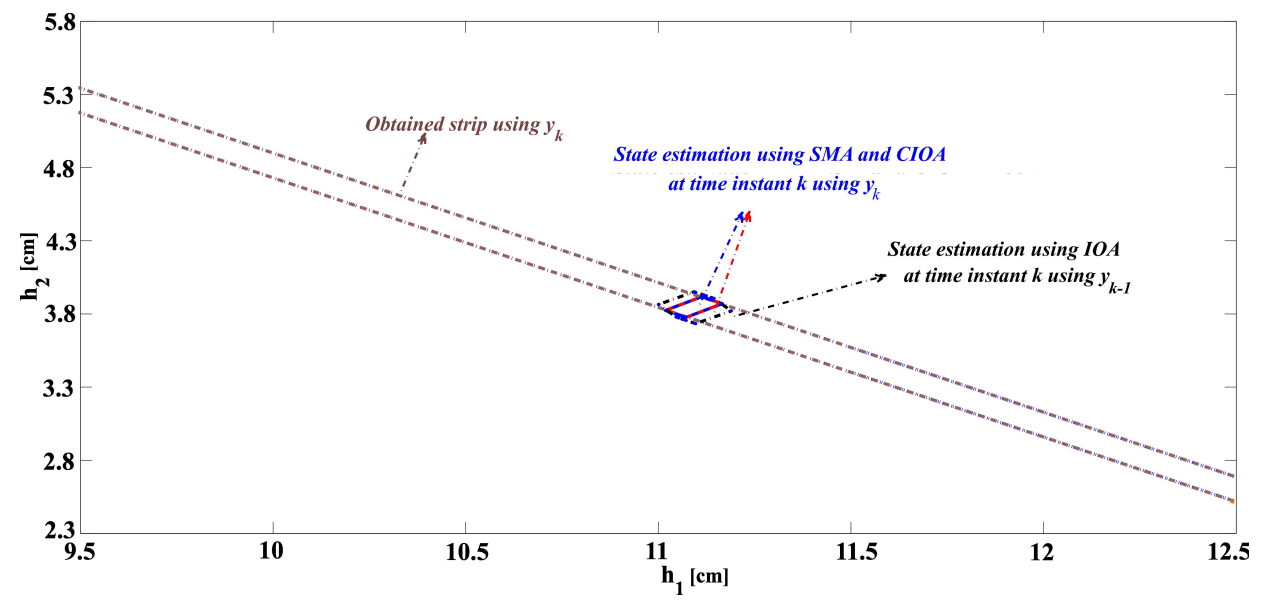

FIGURE 5 State estimation in the state space.

TABLE 1 The constants $L^{\mathrm{io}, *}, L^{\mathrm{cio}, *}$ and $\lambda^{*}$ in steady state.

\begin{tabular}{ccc}
\hline SMA & Standard IOA & CIOA \\
\hline$\lambda_{\infty}^{*}=\left[\begin{array}{l}0.6980 \\
0.6221\end{array}\right]$ & $L_{\infty}^{\mathrm{io}, *}=\left[\begin{array}{l}0.6980 \\
0.6221\end{array}\right]$ & $L_{\infty}^{\mathrm{cio}, *}=\left[\begin{array}{l}0.6980 \\
0.6221\end{array}\right]$ \\
\hline
\end{tabular}

value of the parameter $\lambda$ is determined by 9 when using the SMA. In addition, the observer gain in the case of IOA and the observer gain in the case of CIOA are computed using (13) and (18), respectively.

Further analysis is done during steady-state operation of the system in order to compare the distinct gains using observerbased approaches (i.e., IOA and CIOA) and parameter $\lambda$ in the SMA since the computed $L^{\mathrm{io}, *}, L^{\text {cio,* }}$ and $\lambda^{*}$ are constant when $k$ tends to infinity. Looking at (9), (13) and (18), it can be noted that the values of $\lambda^{*}, L^{\mathrm{io}, *}$ and $L^{\text {cio,* }}$ are independent parameters with respect to the information of the measured output, i.e., these parameters are designed to minimize the size of the statebounding zonotope. Therefore, by using the same initial conditions, the same values of $\lambda^{*}, L^{\mathrm{io}, *}$ and $L^{\mathrm{cio}, *}$ can be obtained in steady state when using (9), (13) and (18). In this regard, Table 1 shows the obtained results during steady-state operation of the system from the simulation of the approaches.

Moreover, the time evaluation of the state estimation can be computed at each time instant. The interval hull of the state estimation is projected into the output space by using the system matrix $C$. Figure 6 is obtained from the simulation as a result of the projection of the state estimation into the output space. Note from Figure 6 at that the maximum and the minimum bounds of the obtained zonotopic state estimations using both CIOA and SMA are the same. But, there is one-time-instant delay between the results obtained using the standard IOA and the other approaches since the information of the output measurement at $k-1$ given $k$ is used in IOA.

In order to emphasize the difference between the IOA and the other approaches, the center of the zonotopic state estimations are projected into the output space and shown in Figure $6 \mathrm{~b}$ It can be observed from Figure $6 \mathrm{~b}$ the center of the zonotopic state estimations are determined using CIOA and SMA approaches are the same.

\section{3 | Application to FD}

In this section, the extension of the comparison of both IOA and SMA approaches is performed when applied to FD. Although, as shown in the previous section, both CIOA and SMA approaches are equivalent when applied to state estimation. But, CIOA cannot be used for FD tasks as discussed in Section 3 Thus, instead of using CIOA this section considers IOA for comparison purposes. The comparison is based on the minimum fault magnitude that can detect the considered approaches. In this regard, the same actuator and sensor fault scenarios as Section 3 are considered in this case study. 


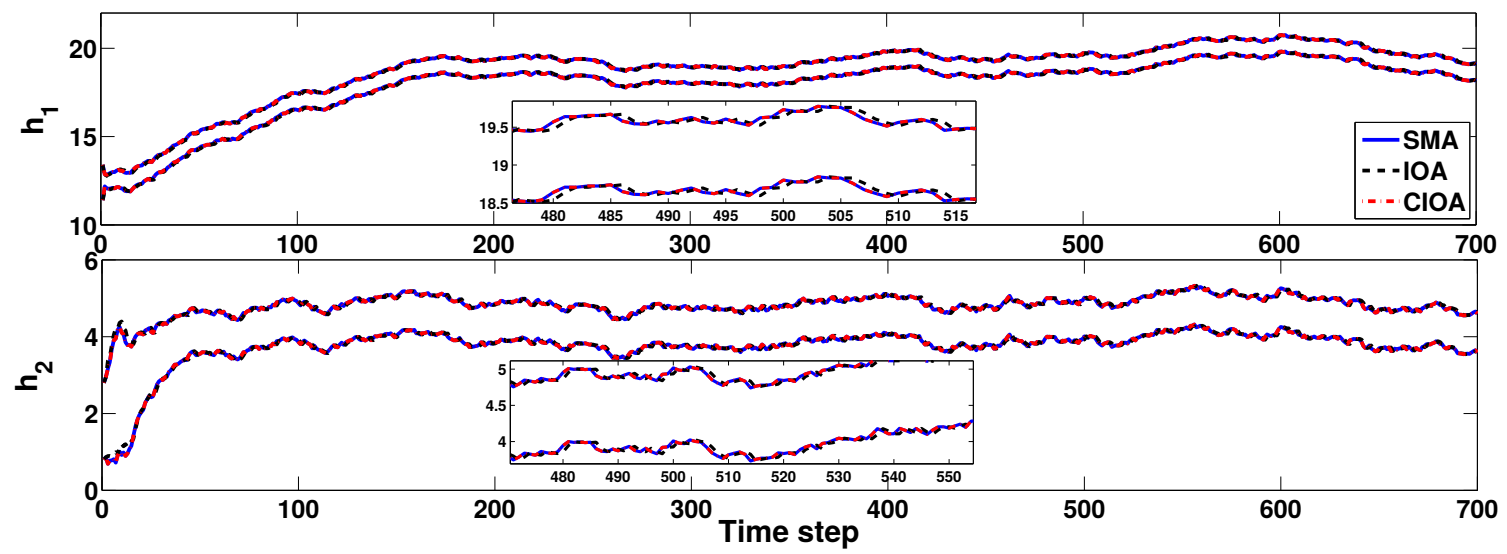

(a) Projection of the state estimation into the output space.

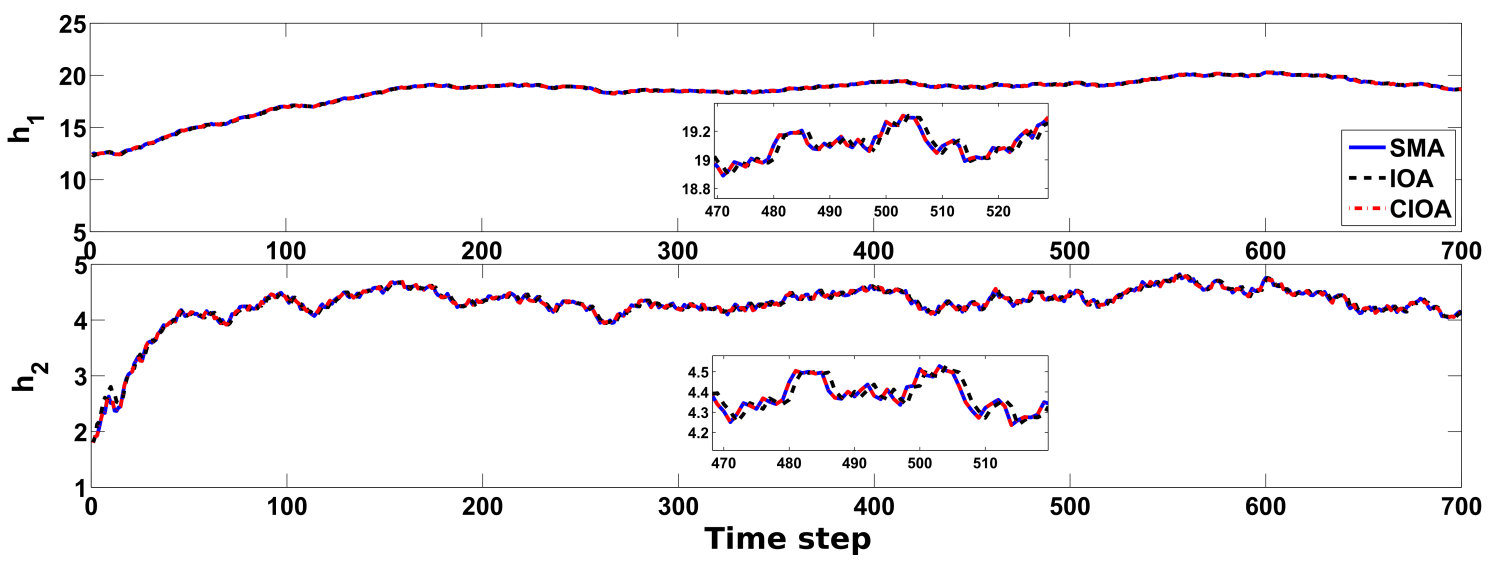

(b) The centers of the projection of the state estimation into the output space.

FIGURE 6 State estimation into the output space.

TABLE 2 Minimum detectable fault during steady state.

\begin{tabular}{cccccc}
\hline & \multicolumn{2}{c}{ Actuator fault } & \multicolumn{2}{c}{ Input sensor fault } & Output sensor fault \\
& $f^{a_{1}}$ & $f^{a_{2}}$ & $f^{u_{1}}$ & $f^{u_{2}}$ & $f^{y_{1}}$ \\
\hline SMA & \pm 0.285 & \pm 0.66 & $\pm 0.2 \mathrm{~V}$ & $\pm 0.04 \mathrm{~V}$ & $\pm 0.7 \mathrm{~V}$ \\
IOA & \pm 0.285 & \pm 0.66 & $\pm 0.2 \mathrm{~V}$ & $\pm 0.04 \mathrm{~V}$ & $\pm 0.7 \mathrm{~V}$ \\
\hline
\end{tabular}

The minimum detectable output sensor, input sensor and actuator faults can be computed using Theorems 4,5 and 6 for the IOA and Theorems 4, 5 and 6 for SMA, respectively. The obtained results are reported in Table 2 all of them evaluated in steady-state regime.

According to Table 2 the minimum magnitude of the sensor and actuator faults able to be detected through the use of both SMA and IOA are identical since the zonotope-shape matrices of the state estimations are also identical when using both approaches in steady state, i.e., $\hat{H}_{\infty}^{\mathrm{x}_{\mathrm{io}}}=H_{\infty}^{\mathrm{x}_{\mathrm{e}}}$. Therefore, as discussed in Section 3 the use of both SMA and IOA results in the same minimum detectable fault magnitudes in steady state.

For the purpose of further analysis, a sensor fault scenario, affecting the output sensor, is considered in whole time range (no only in steady state). Figure 7 shows the minimum magnitude of the output sensor fault that can be detected using both SMA and IOA. Based on the results shown in Figure 7 IOA conservativeness is slightly greater with respect to SMA during the transient regime. However, as already presented in Table 2 and shown in Figure 7 the same minimum detectable fault 


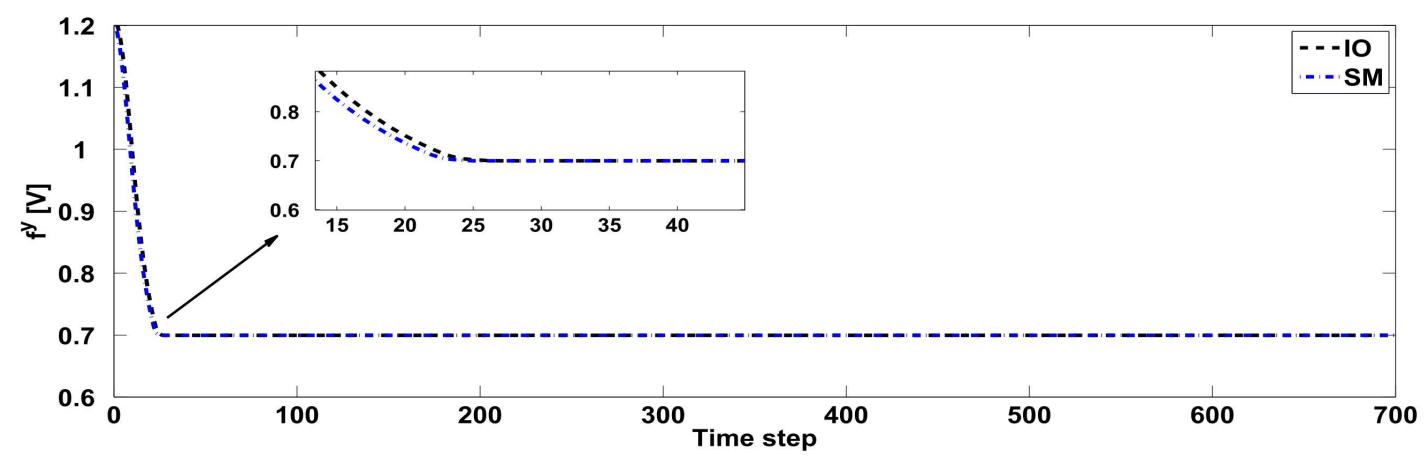

FIGURE 7 Minimum detectable output sensor fault.

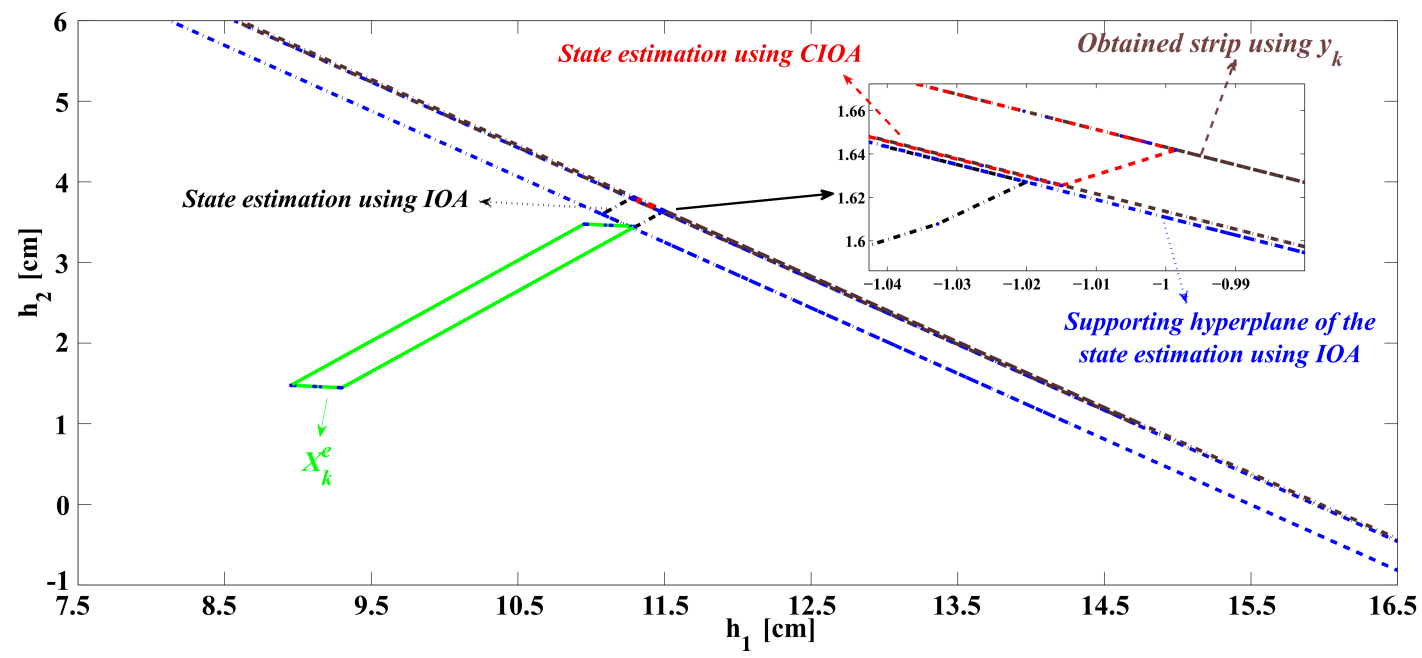

FIGURE 8 Scenario 1: FD results using SMA and IOA in the case $f^{y}=0.8 \mathrm{~V}$.

magnitude is obtained during steady state since $\hat{H}_{\infty}^{\mathrm{x}_{\mathrm{io}}}=H_{\infty}^{\mathrm{x}_{\mathrm{e}}}$. Moreover, Table 2 shows that if the magnitude of the considered fault satisfies $f^{y}>0.7 \mathrm{~V}$, it will be detectable for both SMA and IOA. Otherwise, it will be not detectable by any of both approaches. In the following, this result will be verified in simulation considering two scenarios where the fault appears at time instant $k=200$ and its magnitude changes as follows:

- Scenario 1: Output sensor fault $f^{y}=0.8$, i.e., slightly bigger than 0.7 ,

- Scenario 2: Output sensor fault $f^{y}=0.6$, i.e., slightly smaller than 0.7 .

Figure 8 shows the FD results using both SMA and IOA for Scenario 1 (i.e., when $f^{y}=0.8 \mathrm{~V}$ ). From this figure, it can be observed that both SMA and IOA can detect the fault as it was anticipated in the minimun fault detectability analysis. By comparing Algorithm 2 , which introduces the FD test based on SMA, and Algorithm 3 , which introduces the FD using the strip-based IOA, it can be seen that both approaches use the same information of the measured output for generating the strip. Therefore, the same strips are computed when applied to FD by both approaches as shown in Figure 8 The only difference between IOA and SMA is the use of different state-bounding zonotopes for checking the intersection with the strip obtained by using the measurement. More precisely, in the case of SMA, the state-bounding zonotope is computed using (6) and, in the case of IOA, the state-bounding zonotope is computed using (12) for consistency test.

Figure 8 is obtained at time instant $k=201$ (one time instant after the fault occurrence). That figure shows that the existence of the fault is detected by both SMA and IOA since the empty intersection is obtained between the measurement strip and the zonotopes computed by (6) and (12) for SMA and IOA, respectively. In addition, the state estimation determined by CIOA is also shown in Figure 8 It can be noted that the state estimation using CIOA is affected by the fault based on using the 


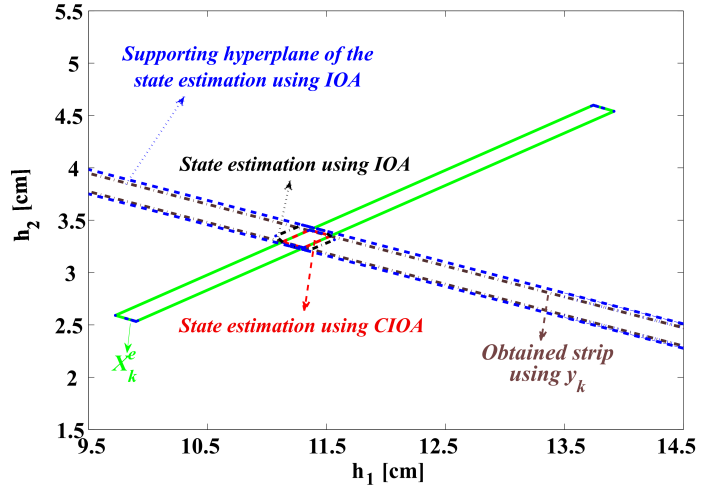

(a) Before occurrence of the fault.

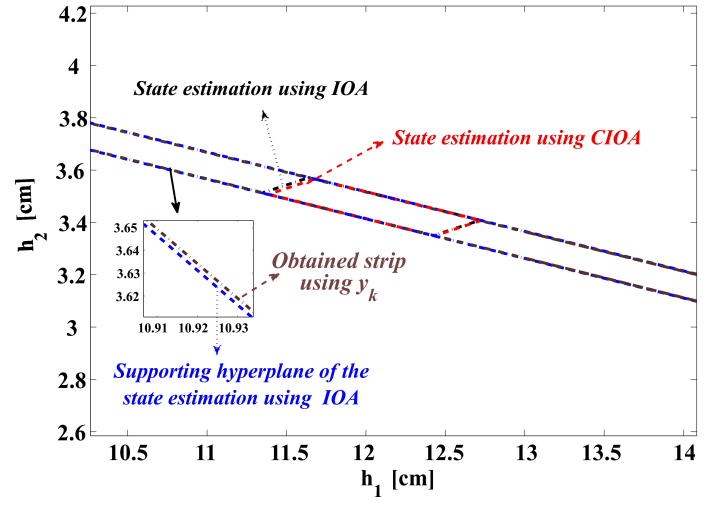

(b) After occurrence of the fault.

FIGURE 9 The FD test using SMA and strip-based IOA in the case $f^{y}=0.8 \mathrm{~V}$ before and after occurrence of the fault.

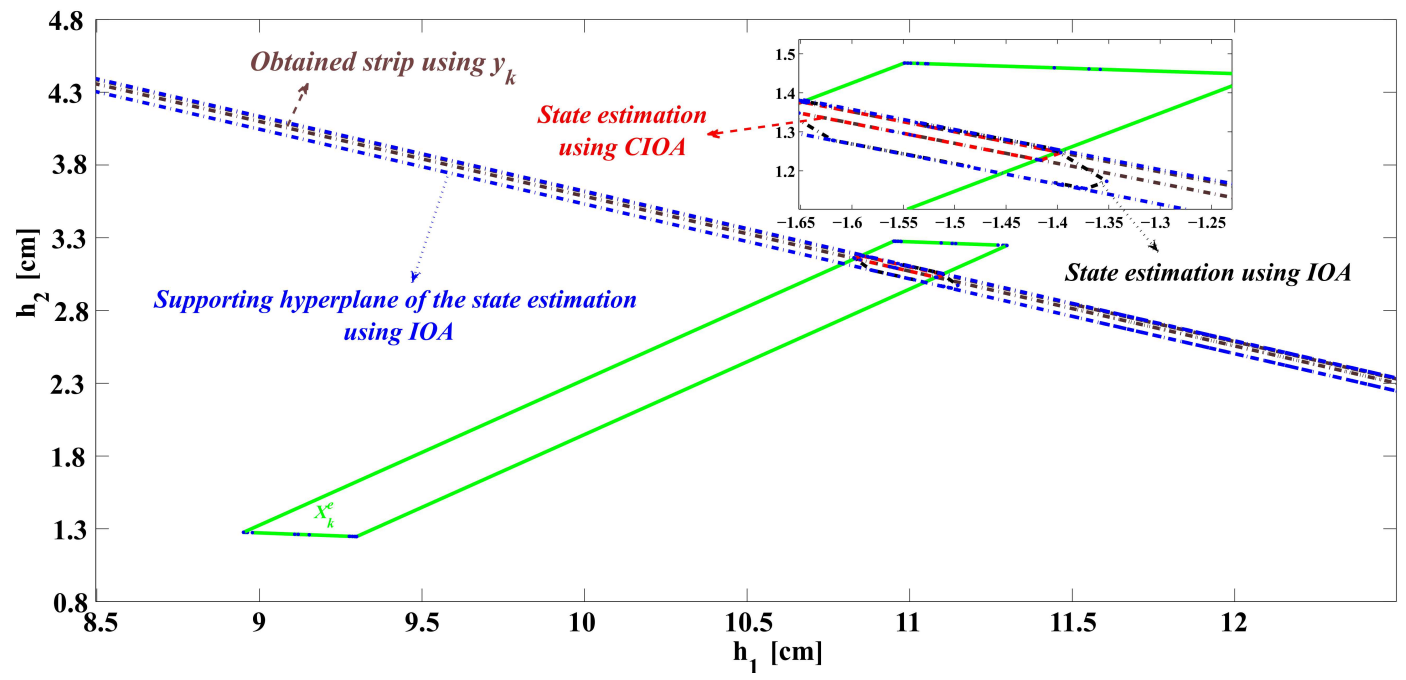

FIGURE 10 Scenario 2: FD results using SMA and IOA in the case $f^{y}=0.6 \mathrm{~V}$.

measurement information from the same time instant of the fault occurrence. Thus, the state estimation set is moved together with the measurement strip. Consequently, the empty intersection that indicates the existence of a fault will never occur using CIOA. For this reason, this approach is not used for FD purposes.

Figure 9 shows the FD results using SMA and IOA before and after the fault occurrence. The existence of the intersection between the strip $\mathcal{X}^{y k}$ and the zonotopes $\mathcal{X}^{\mathrm{e}}$ and $\hat{\mathcal{X}}^{\mathrm{io}}$ can be seen in Figure 9 a. Then, after detecting the fault with both approaches, monitoring the system with SMA should be stopped but the system could still be monitored using IOA as shown in Figure $9 \mathrm{~b}$. Thus, the ability of monitoring the system after the fault occurrence can be considered as an advantage of IOA with respect to using SMA.

Now, the fault detection test results will be presented in the case of Scenario 2, which considers the fault magnitude as $f^{y}=0.6$ $\mathrm{V}$, i.e., satisfying $f^{y}<0.7 \mathrm{~V}$. As shown in Figure 10 the fault cannot be detected by any of the approaches. This figure shows the strip $\mathcal{X}^{y k}$ and the zonotopes $\mathcal{X}^{\mathrm{e}}$ and $\mathcal{X}^{\text {io }}$ at time instant $k=201$. Note that $\mathcal{X}^{y k} \cap \hat{\mathcal{X}}^{\text {io }} \neq \emptyset$ in this case. Then, when using Algorithm 3 , the fault cannot be detected by using IOA. Similarly, using Algorithm 2 since $\mathcal{X}^{y k} \cap \mathcal{X}^{\mathrm{x}_{\mathrm{e}}} \neq \emptyset$, SMA will neither be able to detect the fault. Finally, Figure 11 shows the FD test before and after the occurrence of the fault. This figure shows that both IOA and SMA are not able to determine the existence of the fault in accordance with the results presented in Table 2 


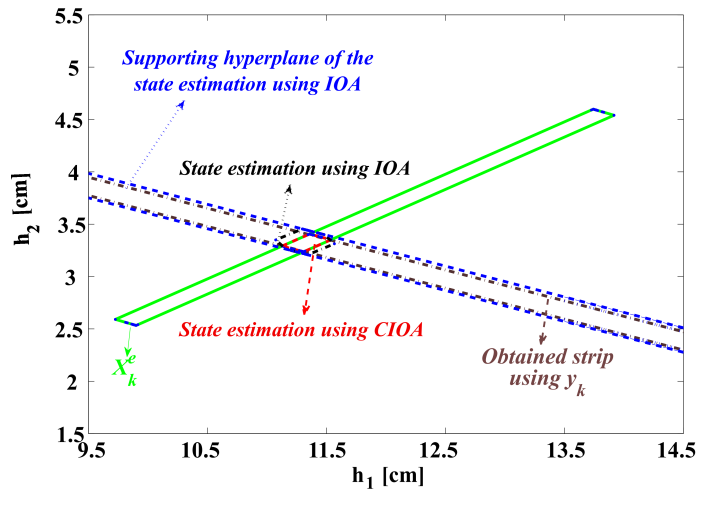

(a) Before occurrence of the fault.

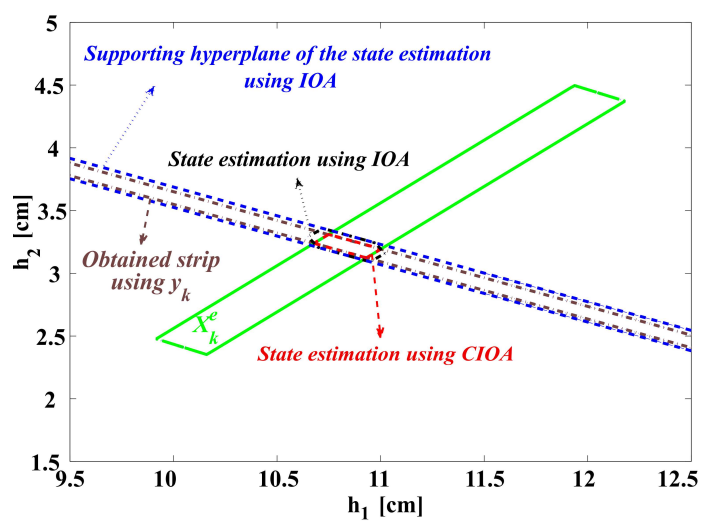

(b) After occurrence of the fault

FIGURE 11 FD test using SMA and strip-based IOA in the case $f^{y}=0.6 \mathrm{~V}$ before and after occurrence of the fault.

\section{5 | CONCLUSIONS}

In this paper, both interval-observer-based (IOA) and set membership approaches (SMA) are considered for both state estimation and fault detection (FD) tasks in uncertain linear systems. Both approaches are analyzed and compared from the mathematical point of view using a zonotopic representation of the involved sets. Mathematical and algorithmic formulations of both approaches are introduced to facilitate a fair comparison. As a result, it has been proved that, in the case that the observer gain in IOA is designed based on a current IOA, the state estimation bounding zonotopes are the same as those obtained using SMA. Furthermore, the application of both IOA and SMA is analyzed when applied to FD tasks for different type of faults. The performance of both approaches are characterized in terms of a minimum detectable fault, allowing also the quantitative comparison. The obtained results show that the use of the strip-based FD test for IOA leads to demonstrate that both IOA and SMA presents comparable performance in state-steady regime. Finally, a two-tanks system is considered as a case study for illustrating the effectiveness of both approaches in state estimation and FD purposes. As a future research, the comparison will be extended to the case of nonlinear systems represented with Takagi-Sugeno or linear parameter-varying (LPV) models to assess the performance of both approaches regarding both fault detection and isolation.

\section{APPENDIX: USEFUL DEFINITIONS}

In the following, some fundamental definitions and properties regarding zonotopes are recalled.

Definition A1: (Minkowski Sum) The Minkowski sum of two sets is defined by $\mathcal{X} \oplus \mathcal{Y}=\{x+y: x \in \mathcal{X}, y \in \mathcal{Y}\}$.

Definition A2: (m-order Zonotope) A zonotope is a convex symmetric polytope. Given a vector $p \in \mathbb{R}^{n}$ and a matrix $H \in$ $\mathbb{R}^{n \times m}(n \leq m)$, the zonotope is represented as $\mathcal{Z}=p \oplus H \mathbf{B}^{m_{z}}=\left\{p+H z: z \in \mathbf{B}^{m_{z}}\right\}$, where $p$ is the center, $H$ contains the segments of the zonotope and $\mathbf{B}^{m_{z}}$ is an $m_{z}$-dimensional unitary box. Also, the order $m_{z}$ is a measure for the geometrical complexity of the zonotopes.

Definition A3: (Strip) A strip is described by $S=\left\{x:\left|c^{T} x-d\right| \leq \sigma\right\}$, where $c \in \mathbb{R}^{n_{y} \times n_{x}}$ is a vector and $d, \sigma \in \mathbb{R}^{n_{y}}$ are scalars.

Definition A4: (Interval Hull) Interval hull of a given zonotope $\mathcal{Z}=p \oplus H \mathbf{B}^{m_{z}}$ is the smallest interval box that contain $\mathcal{Z}$ and it is denoted by $\square \mathcal{Z}$.

Definition A5: (Covariation of a Zonotope) Given a zonotope $\mathcal{Z}=p \oplus H \mathbf{B}^{m_{z}}$, the covariation of this zonotope is defined as $\operatorname{cov}(\mathcal{Z})=H H^{T}$. 
Definition A6: (Matrix Trace) The trace of a square matrix $A_{n \times n}$ is defined to be the sum of the elements of its main diagonal. It is obtained as $\operatorname{tr}(A)=\sum_{i=1}^{n} a_{i i}$. Furthermore, for a matrix $X=\left[X_{i j}\right]$ and, square matrices A and B,

$$
\begin{aligned}
\operatorname{tr}(A) & =\operatorname{tr}\left(A^{T}\right), \\
\operatorname{tr}(A+B) & =\operatorname{tr}(A)+\operatorname{tr}(B), \\
\operatorname{tr}(\alpha A) & =\alpha \operatorname{tr}(A), \\
\partial_{x} \operatorname{tr}\left(A X^{\top} B\right) & =A^{\top} B^{\top}, \\
\partial_{X} \operatorname{tr}\left(A X B X^{\top} C\right) & =B X^{\top} C A+B^{\top} X^{\top} A^{\top} C^{\top},
\end{aligned}
$$

hold, where $\partial_{X} \operatorname{tr}(\cdot)$ is a short notation for $\frac{\partial \operatorname{tr}(\cdot)}{\partial X}$.

Definition A7: (Frobenius Norm) The Frobenius Norm or Euclidean norm of a matrix $A_{m \times n}$ is defined as

$$
\|A\|_{F}=\sqrt{\sum_{i=1}^{m} \sum_{j=1}^{n}\left|a_{i i}\right|^{2}}=\sqrt{\operatorname{tr}\left(A A^{H}\right)}
$$

where $A^{H}$ is the conjugate transpose of matrix $A$.

Definition A9: (F-radius) The F-radius of a given zonotope $\mathcal{Z}=p \oplus H \mathbf{B}^{m_{z}} \in \mathbb{R}^{n}$ is the Frobenius norm of that zonotope, i.e., $\|\mathcal{Z}\|_{F}=\|H\|_{F}$.

Property A1: Given two zonotopes $\mathcal{Z}_{1}=p_{1} \oplus H \mathbf{B}^{m_{z_{1}}} \in \mathbb{R}^{n}$ and $\mathcal{Z}_{2}=p_{1} \oplus H \mathbf{B}^{m_{z_{2}}} \in \mathbb{R}^{n}$, the Minkowski sum of these zonotopes is defined as $\mathcal{Z}=\mathcal{Z}_{1} \oplus \mathcal{Z}_{2}=\left(p_{1}+p_{2} \oplus\left[\begin{array}{ll}H_{1} & H_{2}\end{array}\right] \mathbf{B}^{m_{z_{1}}+m_{z_{2}}}\right.$, that is still a zonotope.

Property A2: Given the zonotope $\mathcal{Z}=p \oplus H \mathbf{B}^{m_{z}} \subset \mathbb{R}^{n}$, the strip $S=\left\{x \in \mathbb{R}^{n}:\left|c^{T} x-d\right| \leq \sigma\right\}$ and the vector $\lambda \in \mathbb{R}^{n}$, the intersection between the zonotope and the strip is defined as $\mathcal{Z} \cap S=\hat{p}(\lambda) \oplus \hat{H}(\lambda) \mathbf{B}^{m_{z}+1}$, where $\hat{p}(\lambda)=p+\lambda(d-c p)$ and $\hat{H}(\lambda)=[(I-\lambda c) H \sigma \lambda]$.

Property A3: (Reduction operator) A reduction operator denoted $\downarrow_{q}$ permits to reduce the number of generators of a zonotope $\mathcal{Z}=p \oplus H \mathbf{B}^{m_{z}} \subset \mathbb{R}^{n}$ to a fixed number $q$ while preserving the inclusion property $\mathcal{Z}=p \oplus \downarrow_{q}\{H\} \mathbf{B}^{m_{z}} \subset \mathbb{R}^{n}$. A simple yet efficient solution to compute $\downarrow_{q}\{H\}$ is given in ${ }^{21 \mid 37]}$. It consists in sorting the columns of $H$ on decreasing Euclidean norm and enclosing the influence of the smaller columns only into an easily computable interval hull, so that the resulting matrix $\downarrow_{q}\{H\}$ has no more than $q$ columns.

\section{ACKNOWLEDGMENTS}

This work has been partially funded by the Spanish State Research Agency (AEI) and the European Regional Development Fund (ERFD) through the projects DEOCS (ref. MINECO DPI2016-76493) and SCAV (ref. MINECO DPI2017-88403-R). This work has also been partially funded by AGAUR of Generalitat de Catalunya through the Advanced Control Systems (SAC) group grant (2017 SGR 482) and by Agència de Gestió d'Ajuts Universitaris i de Recerca.

\section{References}

1. Blanke M, Kinnaert M, Lunze J, Staroswiecki M. Diagnosis and Fault-Tolerant Control. USA: Springer; 2006.

2. Gertler J. Fault detection and diagnosis in engineering systems. USA: CRC Press; 1998.

3. Kalman RE. A new approach to linear filtering and prediction problems. Journal of Basic Engineering. 1960;82(1):35-45.

4. Efimov D, Raïssi T, Chebotarev S, Zolghadri A. Interval state observer for nonlinear time varying systems. Automatica. 2013;49(1):200-205.

5. Mazenc F, Bernard O. Interval observers for linear time-invariant systems with disturbances. Automatica. 2011;47(1):140147.

6. Puig V, Stancu A, Escobet A, Nejjari F, Quevedo J, Patton RJ. Passive robust fault detection using interval observers: Application to the DAMADICS benchmark problem. Control Engineering Practice. 2006;14(6):621 - 633. 
7. Blesa J, Rotondo D, Puig V, Nejjari F. FDI and FTC of wind turbines using the interval observer approach and virtual actuators/sensors. Control Engineering Practice. 2014;24:138-155.

8. Raka SA, Combastel C. Fault detection based on robust adaptive thresholds: A dynamic interval approach. Annual Reviews in Control. 2013;37(1):119-128.

9. Raïssi T, Efimov D, Zolghadri A. Interval state estimation for a class of nonlinear systems. IEEE Transactions on Automatic Control. 2012;57(1):260-265.

10. Alamo T, Bravo JM, Camacho EF. Guaranteed state estimation by zonotopes. Automatica. 2005;41(6):1035-1043.

11. Le VTH, Stoica C, Alamo T, Camacho EF, Dumur D. Zonotope-based set-membership estimation for multi-output uncertain systems. IEEE International Symposium on Intelligent Control. India, 2013;:212-217.

12. Milanese M, Norton J, Piet-Lahanier H, Walter É. Bounding approaches to system identification. USA: Springer Science \& Business Media; 2013.

13. Brondsted A. An introduction to convex polytopes. USA: Springer Science \& Business Media; 2012.

14. Wei G, Liu S, Song Y, Liu Y. Probability-guaranteed set-membership filtering for systems with incomplete measurements. Automatica. 2015;60:12-16.

15. Zhang B, Lam J, Xu S. Reachable set estimation and controller design for distributed delay systems with bounded disturbances. Journal of the Franklin Institute. 2014;351(6):3068-3088.

16. Tornil-Sin S, Ocampo-Martinez C, Puig V, Escobet T. Robust fault detection of non-linear systems using set-membership state estimation based on constraint satisfaction. Engineering Applications of Artificial Intelligence. 2012;25(1):1-10.

17. Yang F, Li Y. Robust set-membership filtering for systems with missing measurement: a linear matrix inequality approach. Signal Processing. 2012;6(4):341-347.

18. Combastel C. A state bounding observer for uncertain non-linear continuous-time systems based on zonotopes. 44th IEEE Conference on Decision and Control and European Control Conference. Spain, 2005;:7228-7234.

19. Xu F, Puig V, Ocampo-Martinez C, Olaru S, Stoican F. Set-theoretic methods in robust detection and isolation of sensor faults. International Journal of Systems Science. 2015;46(13):2317-2334.

20. Le VTH, Alamo T, Camacho EF, Stoica C, Dumur D. A new approach for guaranteed state estimation by zonotopes. 18th World Congress IFAC. Italy, 2011;:9242-9247.

21. Combastel C. A state bounding observer based on zonotopes. European Control Conference. UK, 2003;

22. Chen Jie, Patton Ron J. Robust model-based fault diagnosis for dynamic systems. Springer Science \& Business Media; 2012.

23. Ding Steven. Model-based fault diagnosis techniques: design schemes, algorithms, and tools. Springer Science \& Business Media; 2008.

24. Zhong Maiying, Ding Steven X, Lam James, Wang Haibo. An LMI approach to design robust fault detection filter for uncertain LTI systems. Automatica. 2003;39(3):543-550.

25. Sadrnia MA, Chen J, Patton RJ. Robust fault diagnosis observer design using $H_{\infty}$ optimisation and $\mu$ synthesis. IEE Colloquium on Modelling and Signal Processing for Fault Diagnosis. 1996;:1-9.

26. Liu Nike, Zhou Kemin. Optimal solutions to multi-objective robust fault detection problems. In: :981-988; 2007, USA.

27. Hou M, Patton R J. An LMI approach to $H_{-} / H_{\infty}$ fault detection observers. In: :9305-310; 1996, UK.

28. Li Xiao-Jian, Yang Guang-Hong. Fault detection in finite frequency domain for Takagi-Sugeno fuzzy systems with sensor faults. IEEE Transactions on Cybernetics. 2014;44(8):1446-1458. 
29. Liu Jian, Wang Jian Liang, Yang Guang Hong. An LMI approach to minimum sensitivity analysis with application to fault detection. Automatica. 2005;41(11):1995-2004.

30. Henry David. A norm-based point of view for fault diagnosis. Application to aerospace missions. In: :4-16; 2010, Italy.

31. Jaimoukha Imad M, Li Zhenhai, Papakos Vasilios. A matrix factorization solution to the $H_{-} / H_{\infty}$ fault detection problem. Automatica. 2006;42(11):1907-1912.

32. Wang Jian Liang, Yang Guang-Hong, Liu Jian. An LMI approach to $H_{-}$index and mixed $H_{-} / H_{\infty}$ fault detection observer design. Automatica. 2007;43(9):1656-1665.

33. Ding S X, Jeinsch T, Frank P M, Ding E L. A unified approach to the optimization of fault detection systems. International journal of adaptive control and signal processing. 2000;14(7):725-745.

34. Tabatabaeipour SM, Bak T. Robust observer-based fault estimation and accommodation of discrete-time piecewise linear systems. Journal of the Franklin Institute. 2014;351(1):277-295.

35. Meseguer J, Puig V, Escobet T, Saludes J. Observer gain effect in linear interval observer-based fault detection. Journal of Process Control. 2010;20(8):944-956.

36. Le Vu Tuan Hieu, Stoica Cristina, Alamo Teodoro, Camacho Eduardo F, Dumur Didier. Zonotopes: From guaranteed state-estimation to control. John Wiley \& Sons; 2013.

37. Combastel C. Zonotopes and Kalman observers: Gain optimality under distinct uncertainty paradigms and robust convergence. Automatica. 2015;55:265-273.

38. Ogata K. Discrete-time control systems. USA: Prentice Hall Englewood Cliffs, N. J.; 1995.

39. Le Vu Tuan Hieu, Stoica Cristina, Alamo Teodoro, Camacho Eduardo F, Dumur Didier. Zonotopic guaranteed state estimation for uncertain systems. Automatica. 2013;49(11):3418-3424.

40. Johansson KH. The quadruple-tank process: a multivariable laboratory process with an adjustable zero. IEEE Transactions on Control Systems Technology. 2000;8(3):456-465.

41. Auger F, Hilairet M, Guerrero JM, Monmasson E, Orlowska-Kowalska T, Katsura S. Industrial applications of the Kalman filter: A review. IEEE Transactions on Industrial Electronics. 2013;60(12):5458-5471.

42. Puig Vicenç, Saludes Jordi, Quevedo Joseba. Worst-case simulation of discrete linear time-invariant interval dynamic systems. Reliable Computing. 2003;9(4):251-290.

43. Pourasghar Masoud, Puig Vicenç, Ocampo-Martinez Carlos, Zhang Qinghua. Reduced-order Interval-observer Design for Dynamic Systems with Time-invariant Uncertainty. In: :1-6; 2017.

44. Puig V, Stancu A, Quevedo J. Set versus trajectory based approaches to interval observation. In: ; 2005.

45. Xu F, Puig V, Ocampo-Martinez C, Stoican F, Olaru S. Actuator-fault detection and isolation based on interval observers and invariant sets. 52nd IEEE Annual Conference on Decision and Control. Italy, 2013;:4385-4390.

46. Pourasghar M, Puig V, Ocampo-Martinez C. Characterization of the minimum detectable fault of interval observers by using set-invariance theory. 3rd Conference on Control and Fault-Tolerant Systems. Spain, 2016;:79-86. 